\title{
Magnetohydrodynamics in the Early Universe and the Damping of Non-linear Alfvén Waves
}

\author{
Kandaswamy Subramanian* and John D. Barrow \\ Astronomy Centre, University of Sussex, Brighton BN1 9QH, UK.
}

(July 2, 2018)

\begin{abstract}
The evolution and viscous damping of cosmic magnetic fields in the early universe, is analysed. Using the fact that the fluid, electromagnetic, and shear viscous energy-momentum tensors are all conformally invariant, the evolution is transformed from the expanding universe setting into that in flat spacetime. Particular attention is paid to the evolution of nonlinear Alfvén modes. For a small enough magnetic field, which satisfies our observational constraints, these wave modes either oscillate negligibly or, when they do oscillate, become overdamped. Hence they do not suffer Silk damping on galactic and subgalactic scales. The smallest scale which survives damping depends on the field strength and is of order a dimensionless Alfvén velocity times the usual baryon-photon Silk damping scale. After recombination, nonlinear effects can convert the Alfvén mode into compressional, gravitationally unstable waves and seed cosmic structures if the cosmic magnetic field is sufficiently strong.
\end{abstract}

\section{INTRODUCTION}

The origin of ordered, large-scale cosmic magnetic fields remains a challenging problem. It is widely assumed that magnetic fields in astronomical objects, like galaxies, grew by turbulent dynamo amplification of small seed magnetic fields [1]. There have been many suggestions for producing the small seed magnetic fields required to prime the amplification process [2]. Many of these proposals appeal to processes which might operate in the very early universe [3]. Moreover, the efficiency with which known turbulent dynamo mechanisms can produce the observed fields is still being debated [4]. An alternate possibility is that the galactic field could be the remnant of a larger cosmological field of primordial origin [5], although, as yet, there is no compelling mechanism for producing the required field. It could form part of the initial conditions, arise at a phase transition, or be produced in some way at the end of a period of inflation [3]. If the primordial field is homogeneous then the isotropy of the microwave background places a limit of $3.4 \times 10^{-9}\left(\Omega_{0} h_{50}^{2}\right)^{\frac{1}{2}}$ Gauss on its present strength [6]. This limits the strength of any primordial field on scales greater than the horizon at last scattering.

Dissipative processes play a key role in fashioning the spectrum of irregularities that survive the radiation era. It is well known that density fluctuations in the baryons suf- 
fer "Silk" damping in the early universe, due to radiative viscosity [7]. This leads one to wonder whether fluctuating magnetic fields produced in the early universe, which couple to the baryons, will also be damped by similar processes. This problem was considered by Jedamzik, Katalinic and Olinto [8], who examined the damping of linearised magnetohydrodynamical (MHD) wave modes in an expanding universe. However, there remain subtleties about the formulation of the problem, and the damping processes, which prompt us to reanalyse the problem in a completely different fashion that also allows non-linear effects to be considered.

We are able to do this by exploiting the conformal invariance of the relativistic fluid, electromagnetic, and shear viscous energy-momentum tensors to transform the problem from the expanding universe setting to a simpler problem in flat spacetime. This transformation is explained in detail in the next section and in Appendix A, and it allows us to study the evolution and damping of various MHD waves. After taking the non-relativistic limit of the resulting MHD equations, in Section III, we focus in Section IV on non-linear Alfvén wave solutions and study their damping. The compressible MHD modes are also briefly treated, in their linearised limit, in Section V. As the universe expands, the relevant mean-free-path of the particle responsible for the damping eventually exceeds the wavelength of a given mode. The evolution of various MHD motions in this free-streaming regime, is considered in Section VI. Eventually, the universe recombines to form atoms and the tangles in the magnetic field that survive damping, can create gravitationally unstable perturbations. This post-recombination regime is considered in Section VII. In Section VIII, we connect these ideas together to discuss how a given spectrum of magnetic inhomogeneities evolves, and section IX summarizes our conclusions.

\section{CONFORMAL INVARIANCE OF RELATIVISTIC, VISCOUS MHD}

We shall make use of a conformal invariance property of the field equations of general relativity. Consider two metrics which are related by conformal transformations of the form

$$
g_{\mu \nu}^{*}=\Omega^{2} g_{\mu \nu}
$$

where $\Omega \equiv \Omega\left(x^{\alpha}\right)$. Suppose the cosmic fluid is described by an energy-momentum tensor, say $T^{\mu \nu}$. If this is traceless, $T_{\mu}^{\mu}=0$, then the equations of motion of the fluid are invariant under conformal transformations. That is, we have

$$
T_{; \nu}^{\mu \nu}=0 \quad \Leftrightarrow \quad T_{; * \nu}^{* \mu \nu}=0 \quad \text { with } \quad T^{* \mu \nu}=\Omega^{-6} T^{\mu \nu}
$$

Here ; $*$ denotes a covariant derivative with respect to the metric $g_{\mu \nu}^{*}$. This result is straightforward to demonstrate by direct calculation.

We apply this result to the early universe setting. Firstly, note that the FriedmannRobertson-Walker (FRW) metric is conformally flat. We will only need to consider the zero-curvature metric with line element

$$
d s^{2}=g_{\mu \nu} d x^{\mu} d x^{\nu}=a^{2}(\tau)\left[-d \tau^{2}+d x^{2}+d y^{2}+d z^{2}\right],
$$

where $\tau$ is conformal time and $a(\tau)$ is the scale factor. We also define the comoving proper time $t$ using $d t=a d \tau$. We can relate this metric to flat spacetime, $\eta_{\mu \nu}$, by a conformal 
transformation, $\eta_{\mu \nu}=g_{\mu \nu}^{*}=a^{-2}(\tau) g_{\mu \nu}$, with $\Omega=a^{-1}(\tau)$. So if the energy-momentum tensor of the matter in the early universe is trace-free and transforms under conformal transformations as in Eq. (2.2), then we can transform the fluid equation of motion to a simpler equation in flat spacetime. This approximation method allows us to transform non-linear solutions to the MHD equations, for a fluid with trace-free energy-momentum tensor, from flat spacetime into an expanding FRW universe. We are, of course, neglecting the back-reaction of those motions upon the form of the metric. That is, the gravitational potential (metric) perturbations due to the inhomogeneous motions must be small for this approximation to hold. For example, if the inhomogeneous motions of a radiation fluid give rise to energy density inhomogeneities of amplitude $\delta \rho / \rho$ over a length scale $\lambda$, then the metric perturbations induced are of order $(\delta \rho / \rho)(\lambda / c t)^{2}$. These are small because, for the parameters we consider, $\delta \rho / \rho<<1$ and the perturbations are on sub-horizon scales.

The energy-momentum tensor in the early universe will be modelled by a linear combination of ideal fluid, non-ideal fluid, and electromagnetic fields. We have

$$
T^{\mu \nu}=T_{I}^{\mu \nu}+T_{N I}^{\mu \nu}+T_{E M}^{\mu \nu}
$$

where we have separated the energy-momentum tensor into an ideal fluid part $T_{I}^{\mu \nu}$, a nonideal fluid part $T_{N I}^{\mu \nu}$ and an electromagnetic part $T_{E M}^{\mu \nu}$. We take the ideal part to be a perfect fluid with equation of state $p=\rho / 3$, where $p$ is the pressure and $\rho$ is the energy density (we have set the speed of light $c \equiv 1$.) For most of the period before decoupling, the early universe is radiation dominated and one can use the above equation of state. Later, we will comment on the effects of matter-domination $(p=0)$ before decoupling. Thus, we have

$$
T_{I}^{\mu \nu}=(p+\rho) U^{\mu} U^{\nu}+p g^{\mu \nu}
$$

where $U^{\mu}$ is the normalised four-velocity of the plasma, satisfying the condition $U^{\mu} U_{\mu}=-1$. Note that for $p=\rho / 3$, we have $T_{I \mu}^{\mu}=0$. Under a conformal transformation, the components of $T_{I}^{\mu \nu}$ transform to a new set of (starred) variables as follows

$$
p^{*}=\Omega^{-4} p, \quad \rho^{*}=\Omega^{-4} \rho, \quad U^{* \mu}=\Omega^{-1} U^{\mu}
$$

and the ideal fluid energy-momentum tensor transforms as $T_{I}^{* \mu \nu}=\Omega^{-6} T_{I}^{\mu \nu}$.

The non-ideal fluid part of the energy-momentum tensor can be written as [9]

$$
T_{N I}^{\mu \nu}=-\eta H^{\mu \alpha} H^{\nu \beta} W_{\alpha \beta},
$$

where

$$
H^{\alpha \beta} \equiv g^{\alpha \beta}+U^{\alpha} U^{\beta} ; \quad W_{\alpha \beta} \equiv U_{\alpha ; \beta}+U_{\beta ; \alpha}-\frac{2}{3} g_{\alpha \beta} U_{; \gamma}^{\gamma}
$$

and $\eta$ is the effective shear viscosity coefficient, given by (see Weinberg [9]);

$$
\eta=\frac{4}{15} g \frac{\pi^{2}}{30} T^{4} l_{d}
$$

where $T$ is the temperature of the radiation-dominated universe, $g$ the statistical weight and $l_{d}$ the mean-free-path of the diffusing particle. In the radiation-dominated epoch, the bulk 
viscosity is zero and we have neglected the thermal conductivity term since it does not affect the Alfvén wave mode that we will focus upon.

One can easily check that the trace $T_{N I \mu}^{\mu}=0$. Also, under conformal transformation, using $U^{* \alpha}=\Omega^{-1} U^{\alpha}$ and $U_{\alpha}^{*}=\Omega U_{\alpha}$, we have

$$
W_{\alpha \beta}^{*}=\Omega W_{\alpha \beta}-\left[U_{\alpha} \frac{\partial \Omega}{\partial x^{\beta}}+U_{\beta} \frac{\partial \Omega}{\partial x^{\alpha}}\right]
$$

and $H^{* \mu \alpha} H^{* \nu \beta}=\Omega^{-4} H^{\mu \alpha} H^{\nu \beta}$; so the non-ideal stress conformally transforms as

$$
T_{N I}^{* \mu \nu}=-\eta^{*} \Omega^{-3} H^{\mu \alpha} H^{\nu \beta} W_{\alpha \beta}=\Omega^{-6} T_{N I}^{\mu \nu}, \quad \text { if } \quad \eta^{*} \equiv \Omega^{-3} \eta .
$$

The electromagnetic part of the energy-momentum tensor is given by

$$
T_{E M}^{\mu \nu}=\frac{1}{4 \pi}\left[F_{\gamma}^{\mu} F^{\nu \gamma}-\frac{1}{4} g^{\mu \nu} F_{\gamma \delta} F^{\gamma \delta}\right]
$$

where $F_{\mu \nu} \equiv A_{\nu ; \mu}-A_{\mu ; \nu}=A_{\nu, \mu}-A_{\mu, \nu}$ is the Maxwell tensor, $A_{\mu}$ the four-potential, and a comma denotes an ordinary derivative. This is also traceless: $T_{E M \mu}^{\mu}=0$. The evolution of the electromagnetic field is governed by the Maxwell equations

$$
F_{; \nu}^{\mu \nu}=4 \pi J^{\mu}, \quad F_{[\mu \nu, \gamma]}=0 .
$$

Here, $J^{\mu}$ is the four-current density. Under a conformal transformation, these equations are also invariant provided we transform the electromagnetic field tensor and current density as follows:

$$
F^{* \mu \nu}=\Omega^{-4} F^{\mu \nu} ; \quad J^{* \mu}=\Omega^{-4} J^{\mu} .
$$

Under these transformations we verify that

$$
T_{E M}^{* \mu \nu}=\Omega^{-6} T_{E M}^{\mu \nu} .
$$

Finally, consider the relativistic generalisation of Ohm's law,

$$
J^{\mu}+J^{\nu} U_{\nu} U^{\mu}=\sigma F^{\mu \nu} U_{\nu}
$$

where $\sigma$ is the conductivity of the fluid, measured in the fluid rest frame. Transforming the four-current and velocity to the starred frame, we can see that Ohm's law also remains invariant under conformal transformations if we define a starred conductivity, $\sigma^{*}=\Omega^{-1} \sigma$.

¿From these preliminaries, we see that the total energy-momentum tensor consisting of an ideal fluid, non-ideal fluid, and electromagnetic field is trace-free, and also transforms as in Eq. (2.2) under a conformal transformation. Suppose we choose $\Omega=a^{-1}(\tau)$, with the scale factor normalised such that $a\left(\tau_{0}\right)=1$ at present conformal time $\tau_{0}$. Define a new set of starred variables as in Eqs. (2.6), (2.11) and (2.14). The evolution equations of the starred variables then become,

$$
\begin{aligned}
& T_{, \nu}^{* \mu \nu}=T_{I}^{* \mu \nu}{ }_{, \nu}+T_{N I}^{* \mu \nu}{ }_{, \nu}+T_{E M}^{* \mu \nu}{ }_{, \nu}=0 \\
& F^{* \mu \nu}{ }_{, \nu}=4 \pi J^{* \mu}, \quad F_{[\mu \nu, \gamma]}^{*}=0 \\
& J^{* \mu}+J^{* \nu} U_{\nu}^{*} U^{* \mu}=\sigma^{*} F^{* \mu \nu} U_{\nu}^{*}
\end{aligned}
$$


Since these equations are simply the flat spacetime equations of relativistic, viscous MHD, we can carry over all results obtained in the flat spacetime context to MHD in FRW spacetime on scales such that the metric perturbations created by the magnetic inhomogeneities and their resulting motions are small. The only extra complication introduced by cosmology is the time-variability of the viscosity and conductivity coefficients. (The transformation of MHD equations from the FRW universe to flat space, has also been explicitly constructed in ref. ( [10]). However, they did not include the viscous stress, nor exploit the simple conformal invariance properties stressed here. The idea that flat space solutions can be taken over to FRW universe in the radiation era, has also been used by Liang [11], to study shock waves in the radiation era; but, without the inclusion of magnetic fields).

At this stage, it is useful to point out that any particle species which does not interact with the relativistic fluid is also easy to take into account. Such a non-interacting component may be any species of dark matter, or neutrinos, after they have decoupled from the rest of the matter. The energy-momentum tensor for this component will then be separately conserved. As long as this component perturbs the form of the FRW metric negligibly, the above treatment of conformal transformation of relativistic MHD to flat spacetime will go through. This also means that the MHD equations in the above form can be applied to the baryon-photon-magnetic field system, even after dark matter domination, until the baryon density becomes comparable to the radiation density, which happens close to decoupling. In the sections to follow, we will use these equations to examine the evolution of MHD waves, in particular the non-linear Alfvén mode, after taking the non-relativistic limit of the equations.

\section{NON-RELATIVISTIC LIMIT}

Suppose the universe was seeded with magnetic fields at some early epoch in its radiationdominated phase. We consider the subsequent evolution. For magnetic fields of realistic strengths (ie that are not going to produce highly discordant microwave background fluctuations or radically change the course of primordial nucleosynthesis), the induced bulk fluid velocities are in general highly non-relativistic. Suppose we write the four-velocity in the transformed metric as $U^{* \mu} \equiv(\gamma, \gamma \mathbf{v})$, where $\gamma \equiv\left(1-\mathbf{v}^{2}\right)^{-1 / 2}$. This form satisfies the normalisation condition on the four-velocity and $\mathbf{v}=d \mathbf{x} / d \tau=a d \mathbf{x} / d t$ is exactly the peculiar bulk velocity of the fluid in the FRW metric. The non-relativistic limit corresponds to taking $|\mathbf{v}|<<1$ in the fluid conservation equation in Eq. (2.17) .

If we define the electric field $\mathbf{E}^{*} \equiv\left(E^{* 1}, E^{* 2}, E^{* 3}\right)$ and the magnetic field $\mathbf{B}^{*} \equiv$ $\left(B^{* 1}, B^{* 2}, B^{* 3}\right)$ in the starred metric by

$$
F^{* 0 i}=E^{* i} \quad F^{* 12}=B^{* 3} \quad F^{* 23}=B^{* 1} \quad F^{* 31}=B^{* 2},
$$

then the time component of the fluid equation in Eq. (2.17), in the non-relativistic limit, is

$$
\frac{\partial \rho^{*}}{\partial \tau}+\nabla \cdot\left[\left(\rho^{*}+p^{*}\right) \mathbf{v}\right]-\mathbf{E}^{*} . \mathbf{J}^{*}-\eta^{*} \nabla \cdot \mathbf{f}=0 .
$$

Here, $\mathbf{J}^{*}$ is the current-density vector whose components are the spatial components of the transformed four-current $J^{* \mu}$, and $\mathbf{f}=\nabla\left(\mathbf{v}^{2} / 2\right)-(2 / 3) \mathbf{v} \nabla . \mathbf{v}$. The spatial components give the Euler equation 


$$
\begin{aligned}
\frac{\partial}{\partial \tau}\left[\left(\rho^{*}+p^{*}\right) \mathbf{v}\right]+(\mathbf{v} \cdot \nabla)\left[\left(\rho^{*}+p^{*}\right) \mathbf{v}\right] & +\mathbf{v} \nabla \cdot\left[\left(\rho^{*}+p^{*}\right) \mathbf{v}\right] \\
& =-\nabla p^{*}+\mathbf{J}^{*} \times \mathbf{B}^{*}+\eta^{*}\left[\nabla^{2} \mathbf{v}+\frac{1}{3} \nabla(\nabla \cdot \mathbf{v})\right] .
\end{aligned}
$$

We have assumed here that the net charge density $\left(J^{* 0}\right)$ is negligible.

The Maxwell equations in the starred metric, are

$$
\nabla \times \mathbf{B}^{*}=4 \pi \mathbf{J}^{*}+\frac{\partial \mathbf{E}^{*}}{\partial \tau} \quad \nabla \times \mathbf{E}^{*}=-\frac{\partial \mathbf{B}^{*}}{\partial \tau} \quad \nabla \cdot \mathbf{B}^{*}=0 \quad \nabla \cdot \mathbf{E}^{*}=4 \pi J^{* 0}
$$

They are supplemented by the non-relativistic limit of Ohm's law

$$
\mathbf{E}^{*}+\mathbf{v} \times \mathbf{B}^{*}=\frac{\mathbf{J}^{*}}{\sigma^{*}}
$$

The Maxwell equations in a "Lab frame", with fields E and B defined using the fundamental observers of FRW spacetime, are also easy to write down and are given in Appendix A.

In what follows we shall make the usual assumption that, for non-relativistic velocities, the displacement current term can be neglected. In this case, we can split up the Lorentz force term in the canonical way as $\mathbf{J}^{*} \times \mathbf{B}^{*}=-\nabla \mathbf{B}^{* 2} /(8 \pi)+\left(\mathbf{B}^{*} . \nabla\right)\left(\mathbf{B}^{*} / 4 \pi\right)$. We also assume that the early universe was a perfect conductor (see for example ref [3]) and take the limit of $\sigma \propto \sigma^{*} \rightarrow \infty$, in Ohm's law. The magnetic field then satisfies the ideal limit of the induction equation

$$
\frac{\partial \mathbf{B}^{*}}{\partial \tau}=\nabla \times\left[\mathbf{v} \times \mathbf{B}^{*}\right]
$$

In terms of the "Lab" magnetic field, defined in Appendix A, the ideal-limit induction equation is

$$
\frac{\partial\left(a^{2} \mathbf{B}\right)}{\partial t}=\frac{1}{a} \frac{\partial\left(a^{2} \mathbf{B}\right)}{\partial \tau}=\frac{1}{a} \nabla \times\left[\mathbf{v} \times\left(a^{2} \mathbf{B}\right)\right] .
$$

Let us turn now to consider solutions of the above equations.

Consider first the unperturbed evolution, with a zero peculiar velocity and negligible magnetic fields. The solution of the fluid equations is then $p^{*}=\rho^{*} / 3=C_{1}$ with $C_{1}$ a positive constant. This implies that, in the original variables, $p=\rho / 3=C_{1} / a^{4}$, as expected for the radiation-dominated universe. (Furthermore, for zero peculiar velocity, for any "test" magnetic field, $\mathbf{B}^{*}$ is constant in time, or $\mathbf{B} \propto a^{-2}$, a result which is intuitively expected for the "Lab" magnetic field due to flux freezing in the expanding universe.) Now consider the effect of introducing tangled magnetic fields in the universe. The Lorentz force associated with a tangled field will cause the fluid to move and induce a non-zero peculiar velocity. The coupled system of equations describing the evolution of the velocity and magnetic fields is highly non-linear. For this reason, the authors of ref. [8] examined only the case of weak perturbations around a quasi-uniform field. We shall to begin with, follow a complementary approach and look at special non-linear solutions. This will also give some feel for how a general tangled field configuration will evolve. 


\section{NON-LINEAR ALFVÉN WAVES IN THE EARLY UNIVERSE}

\section{A. The ideal non-viscous regime}

At sufficiently early times, (or, equivalently, for the field and velocity on sufficiently large scales), one may assume the matter is a perfect fluid and neglect any viscous effects. Also, the fluid radiation pressure in the early universe is typically much larger than the magnetic pressure, for the field strengths we are considering. Their ratio is given by

$$
\frac{B^{* 2}}{8 \pi p^{*}}=\frac{B^{2}}{8 \pi p} \approx 3 \times 10^{-7} B_{-9}^{2}
$$

where $B_{-9}$ is the present-day magnetic field in units of $10^{-9}$ Gauss. Here, we have assumed that the fields are simply frozen into the uniformly expanding universe, neglecting the effects of the peculiar velocity. Since $B^{* 2} /\left(8 \pi p^{*}\right)<<1$, to an excellent approximation, one can take the motions induced by the magnetic field to be almost incompressible, with $p^{*}+B^{* 2} /(8 \pi) \approx$ $p^{*}=$ constant and drop the pressure gradient term in the reduced Euler equation (3.3). In this limit we have filtered out "fast" compressible motions and we can look for solutions with $\nabla \cdot \mathbf{v}=0$. Equation (3.2) then gives, in the ideal and non-viscous limit, $\partial \rho^{*} / \partial \tau=0$. Also, the Euler equation reduces to

$$
\frac{\partial \mathbf{v}}{\partial \tau}+(\mathbf{v} \cdot \nabla) \mathbf{v}=\frac{\left(\mathbf{B}^{*} \cdot \nabla\right) \mathbf{B}^{*}}{4 \pi\left(\rho^{*}+p^{*}\right)}
$$

while the induction equation is

$$
\frac{\partial \mathbf{B}^{*}}{\partial \tau}+(\mathbf{v} \cdot \nabla) \mathbf{B}^{*}=\left(\mathbf{B}^{*} \cdot \nabla\right) \mathbf{v}
$$

These equations have a wide class of exact, stable solutions of the form [12]

$$
\mathbf{v}= \pm \frac{\mathbf{B}^{*}}{\sqrt{4 \pi\left(\rho^{*}+p^{*}\right)}}, \quad \frac{\partial \mathbf{v}}{\partial \tau}=0, \quad \nabla \cdot \mathbf{v}=0
$$

In these solutions the nonlinear terms in Eq. (4.2) and (4.3) exactly cancel. Any complicated tangled field pattern is possible, if accompanied by a velocity along the magnetic field at the local Alfvén speed.

A particular case of this solution is one where the magnetic field is split up as $\mathbf{B}^{*}=$ $\mathbf{B}_{0}^{*}+\mathbf{b}^{*}$, into a uniform and constant component, $\mathbf{B}_{0}^{*}$, and a tangled component $\mathbf{b}^{*}(\mathbf{x}, \tau)$. Fix the co-ordinates such that $\mathbf{B}_{0}^{*}$ lies along the $z$-axis, that is $\mathbf{B}_{0}^{*}=B_{0}^{*} \hat{\mathbf{z}}$, where $\hat{\mathbf{z}}$ is the unit vector along $z$. Now choose $\mathbf{v}=\mathbf{b}^{*} /\left(4 \pi\left(\rho^{*}+p^{*}\right)\right)^{1 / 2}$; the nonlinear terms in the Euler and induction equations cancel out and we have

$$
\frac{\partial \mathbf{b}^{*}}{\partial \tau}-V_{A} \frac{\partial \mathbf{b}^{*}}{\partial z}=0, \quad \frac{\partial \mathbf{v}}{\partial \tau}-V_{A} \frac{\partial \mathbf{v}}{\partial z}=0
$$

where we have defined the Alfvén velocity by

$$
V_{A}=\frac{B_{0}^{*}}{\left(4 \pi\left(\rho^{*}+p^{*}\right)\right)^{1 / 2}}=\frac{B}{(4 \pi(\rho+p))^{1 / 2}} \approx 3.8 \times 10^{-4} B_{-9} .
$$


Here, for the numerical estimate, we have taken the dominant contribution to the energy density $\rho=\rho_{\gamma}$, the photon energy density, as would be appropriate in the radiation-dominated era, after the epoch of $e^{+} e^{-}$annihilation. The general solution of Eq.(4.5) is therefore a nonlinear Alfvén wave travelling antiparallel to $\mathbf{B}_{0}^{*}$, with $\mathbf{v}=\mathbf{b}^{*} /\left(4 \pi\left(\rho^{*}+p^{*}\right)\right)^{1 / 2}=$ $\mathbf{F}\left(x, y, z+V_{A} \tau\right)$ with an arbitrary function $\mathbf{F}$. One can also have another class of solutions with $\mathbf{v}=-\mathbf{b}^{*} /\left(4 \pi\left(\rho^{*}+p^{*}\right)\right)^{1 / 2}$, where the wave travels in the same direction as $\mathbf{B}_{0}^{*}$. Both these solutions are stable and they exist as long as the two sets of waves do not overlap in space [12].

It is not possible to generalise these nonlinear solutions to the diffusive viscous regime, in the above form, for arbitrary viscosity and conductivity coefficients. However, there exists a special case (see Landau and Lifshitz [13]), with the velocity and tangled magnetic field having arbitrary strengths, but aligned perpendicular to $\mathbf{B}_{0}^{*}$ and depending only on $z$, where such a generalisation is possible. We now look at this case in more detail.

\section{B. Nonlinear Alfvén waves in the viscous regime}

We begin by reinstating the viscous term in the Euler equation (3.3). As before, assume that the magnetic field can be written as $\mathbf{B}^{*}=\mathbf{B}_{0}^{*}+\mathbf{b}^{*}$, with a uniform $\mathbf{B}_{0}^{*}$. We assume $\mathbf{b}^{*}$ is perpendicular to $\mathbf{B}_{0}^{*}$, but do not put any restriction on the strength of $\mathbf{b}^{*}$ so that it need not be a small perturbation of $\mathbf{B}_{0}^{*}$. We also take the peculiar velocity $\mathbf{v}$ to lie perpendicular to $\mathbf{B}_{0}^{*}$ and assume that all the variables depend only on $z$ and $\tau$. In this case, the velocity perturbation automatically satisfies $\nabla \cdot \mathbf{v}=0$. Further, the ratio of the magnetic energy density to the fluid energy density, $B^{2} /(8 \pi \rho) \sim 10^{-6} B_{-9}^{2}<<1$. So even when there is strong damping of motions induced by the field, and a significant fraction of the field energy density goes into heat, $\rho$ will be perturbed negligibly. It is an excellent approximation to neglect the viscous term in Eq. (3.2). Then, in the ideal limit, Eq. (3.2) implies $\left(\partial \rho^{*} / \partial \tau\right)=0$. The non-linear terms in the Euler and induction equations are individually zero because there is no variation of $\mathbf{b}^{*}$ and $\mathbf{v}$ along the fields. These equations then reduce to

$$
\begin{aligned}
\frac{\partial \mathbf{v}}{\partial \tau}=-\left(\frac{1}{\rho^{*}+p^{*}}\right) \nabla\left[p^{*}+B^{* 2} /(8 \pi)\right] & +\left(\frac{B_{0}^{*}}{4 \pi\left(\rho^{*}+p^{*}\right)}\right) \frac{\partial \mathbf{b}^{*}}{\partial z}+\frac{\eta^{*}}{\left(\rho^{*}+p^{*}\right)} \nabla^{2} \mathbf{v} \\
\frac{\partial \mathbf{b}^{*}}{\partial \tau} & =B_{0}^{*} \frac{\partial \mathbf{v}}{\partial z}
\end{aligned}
$$

Note that the LHS of Eq. (4.7) has zero divergence. The RHS will also have zero divergence only if the total pressure $p *+B^{* 2} /(8 \pi)$ is uniform in space. As mentioned above, this is likely to be a good approximation for this mode since the radiation pressure in the early universe is typically much larger than the magnetic pressure. One can therefore drop the pressure gradient term in the reduced Euler equation (4.7). Writing $\mathbf{b}^{*}=b_{0}(\tau, z) \mathbf{n}$ and $\mathbf{v}=v_{0}(\tau, z) \mathbf{n}$, eliminating $v_{0}$ from Eqns. (4.7) and (4.8), gives a damped wave equation for $b_{0}(\tau, z)$,

$$
\frac{\partial^{2} b_{0}}{\partial \tau^{2}}-\frac{\eta^{*}(\tau)}{\left(\rho^{*}+p^{*}\right)} \frac{\partial}{\partial z^{2}}\left(\frac{\partial b_{0}}{\partial \tau}\right)-V_{A}^{2} \frac{\partial^{2} b_{0}}{\partial z^{2}}=0,
$$


where we have defined the Alfvén velocity, $V_{A}$, as before. This linear equation generalises the nonlinear Alfvén mode to the viscous regime. It can easily be solved by taking a spatial Fourier transform. For any mode $b_{0}(\tau, z)=f(\tau) e^{i k z}$. we have

$$
\ddot{f}+\frac{\eta^{*}(\tau) k^{2}}{\left(\rho^{*}+p^{*}\right)} \dot{f}+k^{2} V_{A}^{2} f=0
$$

which is the equation for a damped harmonic oscillator.

The behaviour of solutions to the damped oscillator equation depends on the relative strengths of the driving and damping terms. Suppose we define

$$
\omega_{0}=k V_{A} ; \quad D=\frac{\eta^{*}(\tau) k^{2}}{\left(\rho^{*}+p^{*}\right)} \propto \tau^{2} .
$$

If $\omega_{0}>>D$, then we will have damped oscillatory motion. In the other extreme limit of $D>>\omega_{0}$, the motion becomes overdamped. While one solution of the second-order differential equation suffers strong damping, the other independent solution is negligibly damped. The physical reason for this is that, under strong friction, any oscillator displaced from equilibrium and released from rest has only to acquire a small "terminal" velocity, so that friction balances the driving force. An oscillator starting from this "phase" of oscillation, will then almost freeze, and the associated energy in the oscillator will decrease negligibly. On the other hand, an oscillator mode with a large initial velocity will be significantly damped by the strong friction. Therefore, it is important to consider the ratio $D / \omega_{0}$ to determine which limit applies for the nonlinear Alfvén modes.

We focus primarily on damping by photon viscosity. This is the most important source of viscosity, after $e^{+} e^{-}$annihilation. Also, it is the dissipative process with the potential to damp the largest scales. (Smaller-scale damping by neutrinos is briefly discussed in Section VII and Appendix C). The radiative viscosity coefficient is given by Eq. (2.9), with $g=g_{\gamma}=2$, and the photon mean-free-path is

$$
l_{\gamma}(\tau)=\frac{1}{\sigma_{T} n_{e}(\tau)} \approx 9.5 \times 10^{21} \mathrm{~cm}\left(\frac{T}{0.25 \mathrm{eV}}\right)^{-3}\left(\frac{\Omega_{b} h^{2}}{0.0125}\right)^{-1} x_{e}^{-1} .
$$

Here, $\sigma_{T}$ is the Thomson cross-section for electron-photon scattering, $n_{e}$ is the electron number density, $x_{e}$ the ionisation fraction, and $\Omega_{b}$ is the baryon density of the universe $\rho_{b}$, in units of the closure density. (For later convenience we define the quantity $f_{b} \equiv$ $\left(\Omega_{b} / 0.0125 h^{-2}\right)$, which measures the baryon density, in terms of the preferred value given by Walker et al. [14]. This used to be the canonical value determined from nucleosynthesis constraints, although at present there is some debate on this issue [15]). In the early universe, the energy density of the baryon-photon fluid is dominated by the photon energy, density $\rho_{\gamma}$; so, using $p^{*}=\rho^{*} / 3, \rho=\rho^{*} / a^{4}, \eta^{*}=a^{3} \eta$, we have for the damping coefficient

$$
D=\frac{\eta^{*} k^{2}}{\left(\rho^{*}+p^{*}\right)}=a^{3} \frac{4}{15} \frac{\rho_{\gamma}(\tau) l_{\gamma}(\tau) k^{2}}{\left(4 \rho_{\gamma}(\tau) a^{4} / 3\right)}=\frac{1}{5} k^{2} l_{\gamma}\left(\tau_{0}\right) a^{2}(\tau),
$$

where we have used the fact that $l_{\gamma}(\tau) \propto n_{e}^{-1}(\tau) \propto a^{3}(\tau)$ and, as before, $\tau_{0}$ is the conformal time today. Hence, the damping-to-driving ratio is 


$$
\begin{aligned}
\frac{D}{\omega_{0}} & =\frac{\eta^{*} k^{2}}{k V_{A}\left(\rho^{*}+p^{*}\right)}=\frac{1}{5} \frac{k_{p}(\tau) l_{\gamma}(\tau)}{V_{A}} \\
& \approx 526.3 \frac{k_{p}(\tau) l_{\gamma}(\tau)}{B_{-9}} .
\end{aligned}
$$

We have defined the proper wavenumber $k_{p}(\tau)=(k / a(\tau))$, of a Fourier component and substituted for the Alfvén velocity in terms of the field strength using $V_{A} \approx 3.8 \times 10^{-4} B_{-9}$. For the diffusion approximation to be valid, we require $k_{p} l_{\gamma}<1$; that is, we must consider only wavelengths larger than the mean-free-path. Nevertheless, one expects a large range of wavelengths for which modes will fall in the overdamped regime.

In order to consider the evolution of modes of different wavelengths, one has first to look at the quantitative solution of Eq. (4.10). For this, substituting

$$
f(\tau)=\exp \left(-\int \frac{D(\tau)}{2} d \tau\right) W(\tau)
$$

into Eq. (4.10), the evolution of $W$ is given by

$$
\ddot{W}+p(\tau) W=0 \quad \text { with } \quad p(\tau)=\omega_{0}^{2}-\frac{\dot{D}}{2}-\left(\frac{D}{2}\right)^{2} .
$$

When $\omega_{0}>>D$, we have $p \approx \omega_{0}^{2}$ in Eq. (4.16) and then the solution is $W=\exp \left( \pm i \omega_{0} \tau\right)$. Therefore, in the oscillatory limit we have

$$
b_{0}(\tau, z)=\exp \left(-\int \frac{D(\tau)}{2} d \tau\right) e^{ \pm i \omega_{0} \tau+i k z}, \quad \omega_{0}>>D .
$$

In the opposite limit, $D>>\omega_{0}$, we have to solve the oscillator with a time-dependent friction coefficient. One can obtain an approximate WKBJ solution,

$$
W(\tau)=\frac{1}{(-p)^{1 / 4}} \exp \left[ \pm \int(-p)^{1 / 2}(\tau) d \tau\right]
$$

This solution is valid as long as $p(\tau)$ does not vary too rapidly. In the overdamped regime, this condition can be shown to be equivalent to neglecting $\dot{D}$ compared to $D^{2}$. In the limit $\dot{D}<<D^{2}$, the two solutions are given by

$$
f(\tau)=A_{0} \frac{1}{D^{1 / 2}} \exp \left(-\int^{\tau} D\left(\tau^{\prime}\right) d \tau^{\prime}\right) ; \quad f(\tau)=B_{0} \frac{1}{D^{1 / 2}} \exp \left(-\int^{\tau} \frac{\omega_{0}^{2}}{D\left(\tau^{\prime}\right)} d \tau^{\prime}\right)
$$

As advertised, in the overdamped limit, one solution (the $A_{0}$ - mode) is strongly damped while the other solution (with $B_{0} \neq 0$ ) is weakly damped.

For damping by photon viscosity, we have $D \propto a^{2} \propto\left(\tau / \tau_{0}\right)^{2}$ in the radiation-dominated epoch. It is more useful to consider an alternate treatment to that of the WKBJ solution, for the rapidly varying, strongly overdamped regime. One notes that, as the damping increases with time to $D>>\omega_{0}, \dot{f}$ will tend to adjust itself so that the acceleration vanishes, so $\ddot{f} \approx 0$. For example, consider initially the case $f>0, \dot{f}<0$ and $D \dot{f}>\omega_{0}^{2} f$. Then $\ddot{f}>0$ and so the magnitude of $\dot{f}$ decreases (while remaining negative) until we have $D \dot{f}=-\omega_{0}^{2} f$, when 
the acceleration vanishes. On the other hand, if $D \dot{f}<\omega_{0}^{2} f$, then $\ddot{f}<0$ and the magnitude of $\dot{f}$ increases until we have $\ddot{f}=0$. Subsequently as $D$ keeps increasing, $\dot{f}$ can continue to adjust itself to maintain zero $\ddot{f}$. One can argue similarly for all other cases. Therefore, it seems plausible to consider an alternate approximation for the overdamped case, whereby, after the time when $\ddot{f}$ first vanishes, $f$ satisfies the equation

$$
D \dot{f}+\omega_{0}^{2} f=0 ; \quad D>>\omega_{0} .
$$

We will refer to this approximation as the terminal-velocity approximation. The solution in the overdamped regime, under the terminal-velocity approximation, is simply given by

$$
f(\tau)=f\left(\tau_{T}\right) \exp \left(-\int_{\tau_{T}}^{\tau} \frac{\omega_{0}^{2}}{D\left(\tau^{\prime}\right)} d \tau^{\prime}\right) .
$$

Here, $\tau_{T}$ is the conformal time when the mode reaches the terminal-velocity regime, or when the acceleration, $\ddot{f}$, first vanishes. As we explained earlier, for an oscillator with an initial phase such that $\dot{f}$ is already large, this implies strong damping by the time the terminalvelocity regime is reached. On the other hand, for an oscillator which starts from rest, $\dot{f}$ will have to increase negligibly for the $D>>\omega_{0}$ regime, so that $\ddot{f}$ vanishes and Eq. (4.21) applies.

We now move from the study of the non-linear Alfvén modes to consider the damping of all the different MHD modes in their linearised limit. This has already been studied by JKO [8]. However, we shall do this using the formalism developed here to bring out the links with the non-linear situation. We will return to the further evolution of the non-linear Alfvén mode in Section VI.

\section{DAMPING OF LINEARISED COMPRESSIBLE MHD WAVES}

Let us begin with the linearised MHD equations describing small perturbations to density $\rho_{1}^{*}=\rho^{*}-\rho_{0}^{*}$, pressure $p_{1}^{*}=p^{*}-p_{0}^{*}$, and magnetic field $\mathbf{b}^{*}=\mathbf{B}^{*}-\mathbf{B}_{0}^{*}$. We have

$$
\begin{gathered}
\frac{\partial \rho_{1}^{*}}{\partial \tau}+\nabla \cdot\left[\left(\rho_{0}^{*}+p_{0}^{*}\right) \mathbf{v}\right]=0 \\
\frac{\partial}{\partial \tau}\left[\left(\rho_{0}^{*}+p_{0}^{*}\right) \mathbf{v}\right]=-\nabla p_{1}^{*}+\frac{\left[\nabla \times \mathbf{b}^{*}\right] \times \mathbf{B}_{0}^{*}}{4 \pi}+\eta^{*}\left[\nabla^{2} \mathbf{v}+\frac{1}{3} \nabla(\nabla \cdot \mathbf{v})\right] . \\
\frac{\partial \mathbf{b}^{*}}{\partial \tau}=\nabla \times\left[\mathbf{v} \times \mathbf{B}_{0}^{*}\right] \\
\nabla \cdot \mathbf{b}^{*}=0, \quad p_{0}^{*}=\frac{\rho_{0}^{*}}{3}, \quad p_{1}^{*}=\frac{\rho_{1}^{*}}{3}
\end{gathered}
$$

Suppose the perturbation is described in terms of the perturbed comoving position $\delta \mathbf{x}=$ $\xi(\mathbf{x}, \tau)$. The perturbed velocity $\mathbf{v}=(\partial \xi / \partial \tau) \equiv \dot{\xi}$. An integration of the perturbed continuity equation (5.1) and induction equation (5.3) then gives 


$$
\rho_{1}^{*}=-\frac{4}{3} \rho_{0}^{*} \nabla \cdot \xi \quad \mathbf{b}^{*}=\nabla \times\left[\xi \times \mathbf{B}_{0}^{*}\right]
$$

Substituting Eq. (5.5) into the perturbed Euler equation, we get

$$
\ddot{\xi}=\frac{1}{3} \nabla(\nabla . \xi)+\left[\nabla \times\left(\nabla \times\left[\xi \times \mathbf{v}_{A}\right]\right)\right] \times \mathbf{v}_{A}+\frac{3 \eta^{*}}{4 \rho_{0}^{*}}\left[\nabla^{2} \dot{\xi}+\frac{1}{3} \nabla(\nabla . \dot{\xi})\right],
$$

where we have defined $\mathbf{v}_{A}=V_{A} \hat{\mathbf{z}}$. This linear equation describes the evolution and damping of linearised MHD modes in the expanding universe. One can look for plane wave solutions of the form $\xi=\psi(\tau) \exp (i \mathbf{k} \cdot \mathbf{x})$. This leads to a replacement of $\nabla$ by $i \mathbf{k}$ and leads to an evolution equation for the amplitude $\psi$ :

$$
\ddot{\psi}=-\mathbf{k}\left[(\mathbf{k} \cdot \psi)\left[\frac{1}{3}+V_{A}^{2}\right]-V_{A}^{2} k_{z} \psi_{z}\right]-V_{A}^{2} k_{z}^{2} \psi-k_{z}(\mathbf{k} \cdot \psi) V_{A}^{2} \hat{\mathbf{z}}-\frac{3 \eta^{*}}{4 \rho_{0}^{*}}\left[k^{2} \dot{\psi}+\frac{1}{3} \mathbf{k}(\mathbf{k} \cdot \dot{\psi})\right] .
$$

Here a subscript " $z$ " denotes the $z$-component of the relevant quantity. We can now look at various types of solution to the above equation.

First, consider the incompressible mode, with $\nabla \cdot \xi=0$, or $\mathbf{k} \cdot \psi=0$. In this case taking the dot product of Eq. (5.7) with $\mathbf{k}$, we also have $\psi_{z}=0$ (provided $k_{z} \equiv k \cos \theta \neq 0$ ). For this mode, Eq. (5.7) reduces to

$$
\ddot{\psi}+\frac{3 \eta^{*}}{4 \rho_{0}^{*}} k^{2} \dot{\psi}+V_{A}^{2} \cos ^{2} \theta k^{2} \psi=0
$$

This is, as expected, is almost exactly the evolution equation for the Alfvén mode encountered and discussed in detail in the last section. The only difference is that $V_{A}$ is replaced by $V_{A} \cos \theta$, generalising the Alfvén mode propagation to be in a general direction inclined at an angle $\theta$ to the zero-order magnetic field. (Although this generalisation is at the cost of introducing the linear approximation).

The evolution of the compressible modes, can be derived by taking the dot product of Eq. (5.7) with $\mathbf{k}$ and $\hat{\mathbf{z}}$. Defining $A=\mathbf{k} \cdot \psi / k$, we have

$$
\begin{aligned}
& \ddot{A}+\left[\frac{1}{3}+V_{A}^{2}\right] k^{2} A-V_{A}^{2} k^{2} \cos ^{2} \theta \psi_{z}+\frac{\eta^{*}}{\rho_{0}^{*}} k^{2} \dot{A}=0, \\
& \ddot{\psi}_{z}+\frac{1}{3} k^{2} \cos \theta A+\frac{3 \eta^{*}}{4 \rho_{0}^{*}} k^{2} \dot{\psi}_{z}+\frac{\eta^{*}}{4 \rho_{0}^{*}} k^{2} \cos \theta \dot{A}=0 .
\end{aligned}
$$

Consider first the undamped limit with $\eta^{*}=0$. In this case, looking for modes with $A \propto$ $\psi_{z} \propto e^{i \omega \tau}$, we can easily derive the dispersion relations

$$
\frac{\omega^{2}}{k^{2}}=\frac{1}{2}\left[c_{s}^{2}+V_{A}^{2}\right] \pm \frac{1}{2}\left[\left(c_{s}^{2}+V_{A}^{2}\right)^{2}-4 c_{s}^{2} V_{A}^{2} \cos ^{2} \theta\right]^{1 / 2} .
$$

Here, we have defined the sound speed in the relativistic limit $c_{s}=1 / \sqrt{3}$. The plus sign in the above equation corresponds to the fast MHD mode while the negative sign corresponds to the slow mode. In the limit $V_{A}<<c_{s}$, which is generally applicable to our early universe 
context, the dispersion relation for the fast mode becomes $\omega / k \approx c_{s}$, while that of the slow mode becomes $\omega / k \approx V_{A} \cos \theta$.

The general solution of Eq. (5.9) and (5.10) when the damping terms are reinstated is quite complicated to analyze analytically, since it involves a fourth-order differential equation with time-dependent coefficients and the dispersion relation is a fourth-order polynomial. However, we can look at some simple special cases which illustrate the general behaviour.

First, consider the case where $\mathbf{k}$ is parallel to $\mathbf{B}_{0}^{*}$; then $\cos \theta=k_{z} / k=1, A=\psi_{z}$, and the equation for $A$ reduces to

$$
\ddot{A}+\frac{\eta^{*}}{\rho_{0}^{*}} k^{2} \dot{A}+c_{s}^{2} k^{2} A=0 .
$$

This describes a damped sound wave, well studied in the literature in connection with the Silk damping of acoustic baryon-photon fluctuations. Therefore we only look at it briefly, to estimate the Silk damping scale. Because the sound-wave oscillation frequency is such that $\omega_{s}=k c_{s}>>k V_{A}$, these modes do not become overdamped in general, and the damped oscillatory solutions of Section IV can be used to describe their evolution. Specifically, we have

$$
A(\tau)=\exp \left(-\int \frac{D_{s}(\tau)}{2} d \tau\right) e^{ \pm i \omega_{s} \tau}, \quad \omega_{s}>>D_{s}
$$

where $D_{s}=k^{2}\left(\eta^{*} / \rho_{0}^{*}\right)$. These modes get damped by a factor

$$
\exp \left(-\int \frac{D_{s}(\tau)}{2} d \tau\right)=\exp \left[-\frac{k^{2}}{k_{D}^{2}}\right] ; \quad \text { where } \quad k_{D}^{-2}=\frac{2}{15} \int \frac{l_{\gamma} d t}{a^{2}(t)} .
$$

This agrees quite well with the Silk damping of sound waves in the radiation era, derived in more detailed treatments (cf. [17], [20]), in the appropriate limit. In fact, the more detailed derivation of Silk damping, using a Boltzmann treatment gives a damping factor $\exp \left[-\left(k^{2} / k_{D, b o l}^{2}\right)\right]$, where

$$
k_{D, b o l}^{-2}=\frac{2}{15} \int \frac{l_{\gamma} d t}{a^{2}(t)}\left[\frac{(1+R) f_{2}^{-1}+5 R^{2} / 4}{(1+R)^{2}}\right] .
$$

Here, $R=3 \rho_{b} / 4 \rho_{\gamma}$ and $f_{2}=3 / 4$ if the effects of polarisation are included and $f_{2}=1$ otherwise. Notice that, in the limit $R<<1$ applicable to epochs where the radiation density dominates baryon density, and $f_{2}=1$, the damping scales exactly match, with $k_{D}=k_{D, \text { bol }}$. The effects of non-zero $R$ constitute at most about a $20-25 \%$ correction to the damping scale we derive. In the radiation-dominated epoch one has $k_{D}^{-1}=(4 / 45)^{1 / 2} L_{S}(t) / a(t) \sim$ $0.3 L_{S}(t) / a(t)$, where $L_{S}(t)=\left(l_{\gamma} t\right)^{1 / 2}$ is the Silk scale. The largest scales which suffer appreciable damping are the modes with wavelengths $\left(2 \pi k_{D}^{-1}\right)$, of order $L_{S}$ [7].

In the other extreme case, when $\mathbf{k}$ is perpendicular to $\mathbf{B}_{0}^{*}, \cos \theta=k_{z} / k=0$, and the equation for $A$ reduces to

$$
\ddot{A}+\frac{\eta^{*}}{\rho_{0}^{*}} k^{2} \dot{A}+\left[\frac{1}{3}+V_{A}^{2}\right] k^{2} A=0 .
$$


This describes a damped fast-magnetosonic wave. The real part of the oscillation frequency is $\omega_{R}=k\left(c_{s}^{2}+V_{A}^{2}\right)^{1 / 2}>>k V_{A}$, since in general $V_{A}<<c_{s}$. One can see that the damping of these modes is very similar to that of the sound waves (and in fact is exactly the same when we neglect $V_{A}$ compared to $c_{s}$ ). Again, we expect modes with wavelengths less than the Silk scale to be significantly damped.

Now we turn to the damping of modes with arbitrary direction $\mathbf{k}$. In the undamped case, with $\eta^{*}=0$, and when $V_{A}<<c_{s}$, we have seen that the fast mode has the same oscillation frequency as the sound wave and the slow mode as the Alfven wave. This suggests an approximation to capture the damped counterparts of these modes in the limit of weak magnetic field. Let us write the time variation of $A$ and $\psi_{z}$ as $A(\tau) \propto \psi_{z} \propto \exp \left(i \int \omega d \tau\right)$. Suppose $\omega$ is dominated by its real part, and this is of order the undamped frequency of oscillation. We shall later check the consistency of this assumption. Consider first the damped counterpart of the slow mode, in the limit $V_{A}^{2}<<c_{s}^{2}=1 / 3$. For this mode, the ratio of the first two terms in Eq. (5.9), is $\ddot{A} /\left(c_{s}^{2} k^{2} A\right) \sim V_{A}^{2} / c_{s}^{2}<<1$. Also, the ratio of the last term in (5.9) to the second is $\sim D \dot{A} /\left(c_{s}^{2} k^{2} A\right) \sim V_{A} k_{p} l_{\gamma} / 5<<1$. So, these two terms can be neglected when compared to the second term in Eq. (5.9), so $c_{s}^{2} k^{2} A \approx V_{A}^{2} k^{2} \cos \theta \psi_{z}$. For the same reason, one can neglect the last term in Eq. (5.10) compared to the second term in this equation. Substituting $c_{s}^{2} k^{2} A \approx V_{A}^{2} k^{2} \cos \theta \psi_{z}$ in Eq. (5.10), we then have for the damped counterpart of the slow mode,

$$
\ddot{\psi}_{z}+\frac{3 \eta^{*}}{4 \rho_{0}^{*}} k^{2} \dot{\psi}_{z}+V_{A}^{2} k^{2} \cos ^{2} \theta \psi_{z}=0
$$

We see that this is exactly the same equation as that obtained for the damped Alfvén mode, analysed in the last section. Therefore, the slow modes will also be overdamped, and have one solution with negligible damping rate. Our original assumption that $\omega$ is dominated by its real part is valid for this solution, showing the self-consistency of our assumptions. Also, for this mode we have $A \approx\left(V_{A}^{2} / c_{s}^{2}\right) \cos \theta \psi_{z}<<\psi_{z}$, for a general $\theta$; that is, the mode is almost incompressible. (The strongly damped mode has to be analysed differently).

Now consider the fast mode in a similar fashion. We have already derived the exact evolution for the special case when $\cos \theta=0$. Suppose $\cos \theta \neq 0$; assume, as before, that $\omega$ is dominated by its real part, and this is of order the undamped frequency of oscillation. Then, substituting for the time dependence of $A$ and $\psi_{z}$, and taking the real part of Eq. (5.10), we have $\psi_{z} \sim A \cos \theta$. So the ratio of the $\psi_{z}$ term in Eq. (5.9) compared to the second term is $\sim\left(V_{A}^{2} / c_{s}^{2}\right) \cos ^{2} \theta<<1$. Neglecting the $\psi_{z}$ term, and neglecting $V_{A}^{2}$ compared to $c_{s}^{2}$ in Eq. (5.9), results once again in the same equation, Eq. (5.12), for the fast wave as was found for the damped sound waves.

In summary, the above analysis for linear MHD waves, in the limit of weak fields with $V_{A}<<c_{s}$, shows that the fast magnetosonic waves generally damp like sound waves, while there is one mode of the slow magnetosonic wave which behaves exactly as the Alfven mode and gets overdamped. This also agrees with the conclusions reached by JKO.

\section{THE FREE-STREAMING REGIME}

As the universe expands, the mean-free-path of the photon increases as $a^{3}$, while the proper length of any perturbed region increases as $a$. So the photon mean-free-path can 
eventually become larger than the proper wavelength of a given mode. When this happens for any given mode, we will say that the mode has entered the free-streaming regime. Modes with progressively larger wavelengths enter the free-streaming regime up to a proper wavelength $\sim l_{\gamma}\left(T_{d}\right) \sim 10^{22} \mathrm{~cm}$ (see Eq. (4.12) ), or a comoving wavelength of $\sim 3 \mathrm{Mpc}$, at the epoch of decoupling. After (re)combination of electrons and nuclei into atoms, $l_{\gamma}$ increases to a value larger than the present Hubble radius, and all modes enter the free-streaming regime. (We will consider the pre- and post-recombination epochs separately below.)

When photons start to free stream on a given scale of perturbation, the tight-coupling diffusion approximation no longer provides a valid description of the evolution of the perturbed photon-baryon fluid on that scale. One has to integrate the Boltzmann equation for the photons together with the MHD equations for the baryon-magnetic field system. A simpler approximate method of examining the evolution of such modes in the linear regime is to treat the radiation as isotropic and homogeneous, and only consider its frictional damping force on the fluid. (The radiative flux could have also contributed to the force on the baryons; however, for modes with wavelengths smaller than $l_{\gamma}$, this flux is negligible since the associated compressible motions have suffered strong Silk damping at earlier epochs; when the wavelength was larger than $l_{\gamma}$ ). The drag force on the baryon fluid per unit volume due to the radiation energy density $\rho_{\gamma}$, is given by

$$
\mathbf{F}_{D}=-\frac{4}{3} n_{e} \sigma_{T} \rho_{\gamma} \mathbf{v}
$$

Since, typically, less than one electron-photon scattering occurs within a wavelength, the pressure and inertia contributed by the radiation can be neglected when considering the evolution of such modes. The Euler equation for the baryonic component then becomes

$$
\frac{\partial \mathbf{v}}{\partial t}+H(t) \mathbf{v}+\mathbf{v} \cdot \nabla \mathbf{v}=-\frac{1}{a \rho_{b}} \nabla p_{b}+\frac{1}{\rho_{b}} \mathbf{J} \times \mathbf{B}-\frac{1}{a} \nabla \phi-\frac{4 \rho_{\gamma}}{3 \rho_{b}} n_{e} \sigma_{T} \mathbf{v} .
$$

Here, $\rho_{b}$ is the baryon density, $p_{b}$ the fluid pressure, and $H(t)=(d a / d t) / a$ is the Hubble parameter. We have also included the gravitational force, $(1 / a) \nabla \phi$, due to any perturbation in the density. Note that we have written this equation in the unstarred conformal frame, (with the magnetic field defined in the "Lab" frame; see Appendix A), since conformal transformation to flat spacetime is no longer a useful tool in the matter-dominated era. We have also transformed the time co-ordinate, from conformal time, back to "proper time" $d t=a d \tau$.

It should be pointed out that the dramatic drop in the pressure, by a factor of order the very small baryon to photon ratio $\sim 10^{-9}$, when a mode enters the free-streaming regime, has important consequences. First, in the absence of radiation pressure, the effect of magnetic pressure (if it greatly exceeds the fluid pressure) is to convert what was initially an incompressible Alfveń mode into a compressible mode (see below). Second, the effective baryonic Jeans mass decreases dramatically and compressible modes can become gravitationally unstable. Thus, we have to retain the gravitational force term in the above equation. The magnetic pressure will also play a dominant role, providing pressure support against gravity on sufficiently small scales.

The evolution of modes which enter the free-streaming regime depends on the strength of the magnetic fields, in particular whether the magnetic pressure $p_{B}$ is greater or smaller than the fluid pressure, $p_{b}$. For the magnetic pressure, we have 


$$
p_{B}=\frac{B^{2}}{8 \pi}(1+z)^{4} \approx 4 \times 10^{-8} B_{-9}^{2}\left(\frac{1+z}{10^{3}}\right)^{4} \mathrm{dyn} / \mathrm{cm}^{2} .
$$

While the fluid pressure is given by

$$
p_{b}=2 n_{e} k T \approx 1.1 \times 10^{-10}\left(\frac{1+z}{10^{3}}\right)^{4} f_{b} d y n / \mathrm{cm}^{2}
$$

where we have assumed that the fluid temperature is locked to the radiation temperature, and that the gas is an electron-proton gas. By taking the ratio of the two pressures, one can see that magnetic pressure dominates the fluid pressure, (i.e. $p_{B}>>p_{b}$ for $B>>B_{\text {crit }} \sim$ $5 \times 10^{-11}$ Gauss). For magnetic fields smaller than $B_{\text {crit }}$, the fluid pressure dominates.

Consider first the case where the field $B$ is much smaller than $B_{\text {crit }}$. In this case the motions can be assumed incompressible. The Alfvén modes which enter the free-streaming regime, remain Alfvénic. Following the ideas of Section IV, we look again at non-linear Alfvén modes with $\mathbf{B}=\left(\mathbf{B}_{0}+\mathbf{b}\right) / a^{2}$, where $\mathbf{B}_{0}=B_{0} \hat{\mathbf{z}}$, with $B_{0}=$ constant, $\mathbf{b}=\mathbf{n} \bar{b}_{0}(z, t)$ and $\mathbf{v}=\mathbf{n} \bar{v}_{0}(z, t)$, with $\mathbf{n}$ perpendicular to $\hat{\mathbf{z}}$. Recall that $|\mathbf{b}|$ is not necessarily small compared to $\left|\mathbf{B}_{0}\right|$. We assume $\rho_{b}$ to be uniform (but not independent of $t$ ), use the Euler equation (6.2) and the induction equation (3.7), change to conformal time $\tau$, and look for solutions in the form $\bar{b}_{0}(z, t)=\bar{f}(t) e^{i k z}$, following the same procedure as in Section IV. (For the rotational Alfvén-type mode, the gradient terms in (6.2) do not contribute). We obtain an equation for the evolution of $\bar{f}(\tau)$

$$
\frac{d^{2} \bar{f}}{d \tau^{2}}+[a H+\bar{D}] \frac{d \bar{f}}{d \tau}+\bar{\omega}_{0}^{2} \bar{f}=0
$$

where

$$
\bar{\omega}_{0}=k V_{A}\left(\frac{4 \rho_{\gamma}}{3 \rho_{b}}\right)^{1 / 2} ; \quad \bar{D}=n_{e} \sigma_{T} a\left(\frac{4 \rho_{\gamma}}{3 \rho_{b}}\right)
$$

Here, $V_{A}^{2}=3 B_{0}^{2} /\left(16 \pi \rho_{\gamma}\right)=$ constant, is the same as defined in terms of the starred variables in Section IV.

The evolution of this non-linear Alfvén mode depends once again on the relative strengths of the damping and driving terms. The ratio of the viscous damping to expansion damping is given by

$$
\frac{\bar{D}}{a H}=\frac{\left(4 \rho_{\gamma} / 3 \rho_{b}\right) n_{e} \sigma_{T} a}{a H}=\frac{4 \rho_{\gamma}}{3 \rho_{b}} \frac{D_{H}}{l_{\gamma}}>>1
$$

since the Hubble radius $D_{H} \equiv H^{-1}>>l_{\gamma}$, so one can neglect the damping due to Hubble expansion. Also, the ratio of the viscous damping to the driving terms in the oscillator equation $(6.5)$ is

$$
\frac{\bar{D}}{\bar{\omega}_{0}}=\frac{\left(4 \rho_{\gamma} / 3 \rho_{b}\right) n_{e} \sigma_{T} a}{k V_{A}\left(4 \rho_{\gamma} / 3 \rho_{b}\right)^{1 / 2}} \approx 3.04 \times 10^{3}\left(\frac{\rho_{\gamma}}{\rho_{b}}\right)^{1 / 2} \frac{1}{k_{p}(t) l_{\gamma}(t) B_{-9}} .
$$

When a given mode enters the free-streaming limit we will have $k_{p}(t) l_{\gamma}(t) \sim 1$. So, for the field strengths $B_{-9}<\left(B_{\text {crit }} / 10^{-9} G\right)<<1$ that we are considering, all the Alfvén modes are 
strongly overdamped. As the universe expands, the product $k_{p}(t) l_{\gamma}(t) \propto a^{2}$ increases, and at late times any given mode enters the damped oscillatory regime. One can again apply the terminal-velocity approximation of Section IV in the overdamped regime once $d \bar{f} / d \tau$ has adjusted itself to the "zero acceleration" solution. Then, $\bar{f}$ is given by

$$
\bar{f}(\tau)=\bar{C} \exp \left(-\int^{\tau} \frac{\bar{\omega}_{0}^{2}\left(\tau^{\prime}\right)}{\bar{D}\left(\tau^{\prime}\right)} d \tau^{\prime}\right) ; \quad \bar{D}>>\bar{\omega}_{0} .
$$

At sufficiently late times, such that $\bar{D} / \bar{\omega}_{0}<<1$, the damped oscillatory solutions are appropriate, so

$$
\bar{f}=\exp \left(-\int \frac{\bar{D}(\tau)}{2} d \tau\right) e^{ \pm i \bar{\omega}_{0} \tau}, \quad \bar{\omega}_{0}>>\bar{D} .
$$

Now consider the case $B>>B_{\text {crit }}$, when magnetic pressure dominates over the fluid pressure. For $p_{B}>>p_{b}$, incompressibility is no longer a good assumption, and we necessarily will also have gravitationally unstable, magneto-acoustic modes. In general, the pressuregradient term (dominated by the magnetic field) and the magnetic tension are of similar order. The non-uniform magnetic fields (associated with what were initially Alfvén-type modes), can seed compressible motions and their associated density fluctuations. These density fluctuations, can grow by gravitational instability into non-linear structures. Before recombination, there is still significant drag due to the free-streaming photons. But once atoms form, the density of free electrons decreases and the photon mean-free-path becomes larger than the Hubble radius. In the free-streaming regime, the evolution equations of these gravitationally unstable MHD modes are the same before and after recombination. We consider a unified analysis of this evolution in the next section.

\section{THE POST-RECOMBINATION REGIME}

We assume that the perturbations in density and velocity are small enough so that nonlinear terms in the perturbed density and velocity can be neglected. In the Euler equation (6.2), one can neglect the non-linear term, $\mathbf{v} . \nabla \mathbf{v}$ and take the density $\rho_{b}$ to be that of the unperturbed FRW background density. This equation has to be supplemented by the continuity equation for the perturbed fluid density, the Poisson equation for the potential, and the induction equation (3.7). These equations are given explicitly in Appendix B. In the Poisson equation, we take account of the possibility that there may be other forms of collisionless dark matter by writing

$$
\nabla^{2} \phi=4 \pi G a^{2} \delta \rho_{T}=4 \pi G a^{2}\left[\rho_{b} \delta_{b}+\rho_{c} \delta_{c}\right] .
$$

Here, $\delta \rho_{T}$ is the total perturbed density (due to both fluid plus dark matter), $\delta_{b}$ is the fractional perturbation in the fluid, while $\rho_{c}$ and $\delta_{c}$ describe the dark matter density and its fractional perturbation, respectively. We shall adopt the equation of state $p_{b}=2 n_{e} k T=$ $\rho_{b}\left(2 k T / m_{p}\right)=\rho_{b} c_{b}^{2}$, where $m_{p}$ is proton mass.

In general, the magnetic field will be non-uniform when the mode enters the freestreaming regime. We note that, when the background fluid pressure was large,the nonuniform magnetic field may have originally been part of an Alfvén-type incompressible mode. 
However, as we mentioned earlier, once this mode enters the free-streaming regime, there is a dramatic fall in the fluid pressure, by a factor of order the very small baryon to photon ratio $\sim 10^{-9}$. As a result, the pressure of the non-uniform magnetic field, associated with what might well have been an Alfvén-type mode, can no longer be ignored, especially if the field exceeds $B_{\text {crit }}$. (Only a perfectly circularly-polarised Alfvén wave has uniform magnetic pressure). This non-uniform field associated with what started off as an Alfvén-type mode, will now also induce gravitationally unstable, compressible motions.

In treating the resulting evolution, it is usual to assume (cf. Wassermann [16] and Peebles [17]) that perturbations to the Lorentz force, due to that the perturbed velocity, are subdominant with respect to the zeroth-order contribution of the Lorentz force itself. So one takes $\mathbf{B}=\mathbf{B}_{0}(\mathbf{x}) a^{2}$, which solves the induction equation (3.7), if $\mathbf{v}$ is neglected. Of course, this approximation will break down once significant peculiar velocities have been developed, as will always happen on sufficiently small scales, or at sufficiently late times, for any given magnetic field. For galactic scales, it turns out that the distortions to the magnetic field will become important only at late times, even for $B_{-9} \sim 1$. So the above assumption of retaining only the zeroth-order contribution to the Lorentz force is expected to be reasonable. (The equations governing the more general case are derived in Appendix B, and we hope to return to an analysis of this full system elsewhere). Making these assumptions, standard linear perturbation analysis (cf. [16], [17], [18]), leads to the evolution equation for $\delta_{b}$,

$$
\frac{\partial^{2} \delta_{b}}{\partial t^{2}}+\left[2 H+\frac{4 \rho_{\gamma}}{3 \rho_{b}} n_{e} \sigma_{T} a\right] \frac{\partial \delta_{b}}{\partial t}-c_{b}^{2} \nabla^{2} \delta_{b}=4 \pi G a^{2}\left[\rho_{b} \delta_{b}+\rho_{c} \delta_{c}\right]+\frac{1}{a^{3}} S_{0}(\mathbf{x})
$$

where the source term $S_{0}$ is given by

$$
S_{0}=\frac{\nabla \cdot\left[\mathbf{B}_{0} \times\left(\nabla \times \mathbf{B}_{0}\right)\right]}{4 \pi \rho_{b}\left(t_{0}\right)} .
$$

Here, $\rho_{b}\left(t_{0}\right)$ is the fluid density at the present time, $t_{0}$. If we assume the dark matter to be cold, one can also derive a similar equation for its fractional perturbed density $\delta_{c}$. One finds

$$
\frac{\partial^{2} \delta_{c}}{\partial t^{2}}+2 H \frac{\partial \delta_{b}}{\partial t}=4 \pi G a^{2}\left[\rho_{b} \delta_{b}+\rho_{c} \delta_{c}\right]
$$

Prior to recombination, as pointed out in the previous section (Eq. (6.7) ), the viscous damping dominates damping by the Hubble expansion. For $B_{0}>B_{\text {crit }}$, the fluid pressure term can be neglected. Let us also assume that the magnetic field was the only source of initial density perturbations. Then, the compressible modes start their free-streaming evolution with negligible initial $\delta_{b}$ since modes smaller than the Silk scale have been significantly damped and modes on larger scales have a negligible source of pressure perturbations in the radiation era because $\sim 3 V_{A}^{2} / c^{2} \sim 3 \times 10^{-7} B_{-9}$. Thus, Eq. (7.2) can be solved, under the terminal velocity approximation, simply equating viscous damping and the Lorentz force. We obtain

$$
\frac{\partial \delta_{b}}{\partial t}=\frac{S_{0}}{a^{3}}\left(\frac{4 \rho_{\gamma}}{3 \rho_{b}} n_{e} \sigma_{T} a\right)^{-1}=V_{A}^{2} \frac{l_{\gamma}\left(t_{0}\right)}{l_{B}^{2}} \equiv \gamma_{d} .
$$

Here, $l_{B}$ is a typical co-moving coherence scale over which the field varies. Note that the RHS of this equation is constant in time and so the density contrast $\delta_{b}$ increases linearly 
in this epoch. Hence, at the time of recombination, $t_{r}$, the induced baryonic perturbation is $\delta_{b}=\gamma_{d}\left(t_{r}-t_{f}\right)$, where $t_{f}$ is the time when the scale $k_{p}^{-1}(t) \sim l_{B} a(t)$, becomes smaller than the photon mean-free-path. For a flat universe, dominated by dark matter, the total fractional density contrast in the matter is, $\delta=\left(\rho_{b} \delta_{b}+\rho_{c} \delta_{c}\right) /\left(\rho_{b}+\rho_{c}\right) \sim \Omega_{b} \delta_{b}$. At the time of recombination, we then have

$$
\delta\left(t_{r}\right) \approx 3.8 \times 10^{-5} B_{-9}^{2} h^{-3}\left(\frac{l_{B}}{1 M p c}\right)^{-2}\left(1-t_{f} / t_{r}\right) .
$$

On galactic scales with $l_{B} \sim 1 M p c$, we have $t_{f} / t_{r} \sim 0.4$, and then $\delta\left(t_{r}\right) \sim 2.1 \times 10^{-5} h^{-3} B_{-9}^{2}$. This turns out to be small compared to the $\delta$ induced in the post-recombination regime (see below).

Next, consider the post-recombination evolution. The mean-free-path of the photon now increases rapidly to a value exceeding the Hubble radius and viscous damping becomes subdominant compared to expansion damping. Thus, we can neglect the viscous damping term. Also, for $B>B_{\text {crit }}$, we can neglect the fluid pressure term. Now suppose the baryons contribute a fraction $f_{B}$ to the matter density while the cold dark matter contributes a fraction $1-f_{B}$. Then, multiplying Eq. (7.2) by $f_{B}$, (7.4) by $1-f_{B}$, and adding the resulting equations, we get for the total density contrast $\delta=\left(\rho_{b} \delta_{b}+\rho_{c} \delta_{c}\right) /\left(\rho_{b}+\rho_{c}\right)$,

$$
\frac{\partial^{2} \delta}{\partial t^{2}}+2 H \frac{\partial \delta}{\partial t}-4 \pi G a^{2} \rho_{m} \delta=\frac{1}{a^{3}} f_{B} S_{0}(\mathbf{x})
$$

Here, $\rho_{m}$ is the total matter density. Let us assume that at recombination $\delta \approx 0$ and $(\partial \delta / \partial t) \approx 0$; that is, initially there are negligible fluctuations in density and velocity divergence. Note that this is valid for scales much larger than galactic scales. For scales with $l_{B} \sim M p c$, it turns out that, the post recombination evolution induces a $\delta$ much larger than that given in Eq. (7.6), within an expansion time. So including this initial $\delta\left(t_{r}\right)$ gives negligible corrections. The particular solution of (7.7) for a flat matter-dominated universe is given by

$$
\delta(t)=\frac{9}{10} f_{B} t_{0}^{2} S_{0}\left[\left(\frac{t}{t_{r}}\right)^{2 / 3}-\frac{5}{3}+\frac{2}{3}\left(\frac{t}{t_{r}}\right)^{-1}\right] .
$$

This implies that the magnetic field induces a present-day fractional density contrast $\delta_{0}=$ $\delta\left(t_{0}\right)$, with

$$
\delta_{0}=\frac{9}{10} f_{B} t_{0}^{2} S_{0}\left(1+z_{r}\right) \approx \frac{9}{10} f_{B}\left(\frac{V_{A b}\left(t_{0}\right) t_{0}}{l_{B}}\right)^{2}\left(1+z_{r}\right)
$$

where $\left(1+z_{r}\right)=\left(t / t_{r}\right)^{2 / 3}$ and $V_{A b}\left(t_{0}\right)$ is the Alfvén velocity with respect to the baryons given by

$$
\frac{V_{A b}\left(t_{0}\right)}{c}=\frac{B_{0}}{\sqrt{4 \pi \rho_{b}\left(t_{0}\right) c^{2}}} \approx 1.9 \times 10^{-5} B_{-9} f_{b}^{-1 / 2}
$$

Adopting $h=1 / 2,1+z_{r}=1100$, and a flat universe, with $t_{0}=2 /\left(3 H_{0}\right), f_{B}=0.05$, we have 


$$
\delta_{0} \approx 2.96 B_{-9}^{2}\left(\frac{l_{B}}{1 M p c}\right)^{-2} .
$$

We see therefore that a magnetic field with $B_{0} \sim 10^{-9} G$ is needed to impact significantly on galaxy formation. Such fields will also induce rotational perturbations and give significant angular momentum to protogalaxies [16].

¿From Eq. (7.8) and (7.11), it would seem that for sufficiently small, $l_{B}=l_{s}$, say, one can have a $\delta_{b} \sim 1$, even close to recombination. However, the calculation leading to Eq. (7.8) would break down on such small scales because the field distortions induced by the motions, which we have neglected, will become dominant. The resulting magnetic pressures will oppose gravity when the Alfvén crossing time becomes of order the dynamical time; that is, for proper lengths $a(t) l_{B}<l_{J}(t) \sim V_{A b}(t) t$, where $t$ is the relevant dynamical time (the age of the universe) and $l_{J}(t)$ is the magnetic Jeans length. Noting that $V_{A b}(t)=V_{A b}\left(t_{0}\right) a^{-1 / 2}(t)$ and $t=t_{0} a^{3 / 2}$ for a flat universe, the comoving magnetic Jeans length is given by

$$
\lambda_{J}=\frac{l_{J}(t)}{a} \sim V_{A b}\left(t_{0}\right) t_{0} \sim 3.8 \times 10^{-2} B_{-9} h^{-1} f_{b}^{-1 / 2} \mathrm{Mpc} .
$$

Our treatment of how inhomogeneous magnetic fields induce structure formation is valid only for $l_{B}>\lambda_{J}$. On smaller scales one has to solve the full set of equations outlined in Appendix B. We expect strong magneto-sonic waves to be induced by such small scale inhomogeneities in a sufficiently strong magnetic field. These may suffer strong dissipation and so input energy into the IGM. We hope to return to this issue elsewhere.

\section{DISCUSSION}

We have studied the evolution and damping of inhomogeneous magnetic fields in various regimes. It is of interest to synthesise our results and discuss how a given spectrum of magnetic inhomogeneities evolves. We make a few general points and then describe the fate of magnetic inhomogeneities after they enter the Hubble radius.

- As we noted in Section IV, for magnetic field strengths $B_{-9}<1$, the pressure perturbations are negligible. The fast compressible waves induced by these perturbations, have a phase velocity of order the relativistic sound speed, but negligible amplitudes with $\delta_{b} \sim V_{A}^{2} / c_{s}^{2} \sim 3 V_{A}^{2} / c^{2}$. The motions induced by the field can then be treated as incompressible, at least until they enter the free-streaming regime. These slow residual motions occur no faster than the Alfvén timescale, and pressure can constantly readjust on the fast sound-crossing time scale to preserve the incompressibility condition. In this case, Alfvén modes, both non-linear and linear, and the incompressible limit of the slow mode, that we studied in previous sections, are indeed the most relevant.

- It is important to note that linearisation about a constant background field is not a good approximation when following the evolution of magnetic fields which are inhomogeneous with roughly similar power on a multitude of scales. This was one of our motivations for concentrating on the non-linear Alfvén mode, and studying its evolution and damping through various epochs. Although this analysis employed special exact solutions, the amplitude of the tangled component of the magnetic field could be 
taken to be arbitrarily large compared to the amplitude of the large-scale field. Also, its spatial configuration can be arbitrarily specified, by the free function $b_{0}\left(\tau_{0}, z\right)$. So, one expects the behaviour of this mode to reflect, at least qualitatively, the behaviour of general incompressible motions driven by magnetic-field inhomogeneities.

A comoving scale $l_{B}$, which enters the Hubble radius in the radiation-dominated epoch, does so at an epoch $t_{e}$, specified by $l_{B} a\left(t_{e}\right)=2 t_{e}$. Suppose we define this epoch by the radiation temperature $T(t)=T_{0} / a(t)$, where $T_{0}$ is the present-day microwave background radiation temperature, then we have 18

$$
T\left(t_{e}\right)=T_{e}=63 e V\left(\frac{l_{B}}{1 M p c}\right)^{-1} .
$$

In models which produce the field during an inflationary epoch, the initial condition for the non-linear Alfvén wave at the time of horizon entry could be taken to be that the fluid is at rest but the field is tangled. The Lorentz force due to this tangled field will then start pushing on the fluid, when the scale of the tangle becomes smaller than the Hubble radius. On the other hand, if the fields are produced in an early-universe phase transition, they could be associated with large initial velocities.

- In the cosmological context, it should be kept in mind that there is only a finite time for the Alfvén wave to develop and induce motions in the fluid, if they were initially absent. For example, on a comoving scale of $k^{-1}$, by the time of recombination, at $\tau=\tau_{r}$, (or at a temperature $T=T_{r}$ ), the Alfvén wave would have oscillated at most by a phase angle of

$$
\chi=k V_{A}\left(\tau_{r}-\tau_{e}\right)<k V_{A} \tau_{r} \sim 1.8 \times 10^{-1} B_{-9}\left(\frac{k^{-1}}{1 M p c}\right)^{-1}\left(\frac{T_{r}}{0.25 e V}\right)^{-1} .
$$

Here, $\tau_{e}$ is the conformal time when a mode enters the Hubble radius, and we have expressed $\chi$ in terms of the inverse of the wavenumber, $k^{-1}$. Thus, only small-scale magnetic inhomogeneities, with scales $k^{-1}<l_{s} \approx 0.1 B_{-9} M p c$, will have had time to oscillate by more than $\pi / 2$ in phase. Modes on scales $k^{-1}>>l_{s}$, which started with zero initial peculiar velocity, and oscillated by a phase $\chi<<1$ when damping is ignored, cannot damp their tangles, even if damping is included. This situation applies to magnetic fields tangled on galactic scales, with $k^{-1} \sim M p c$. Thus galacticscale magnetic inhomogeneities, with $B_{-9}<1$, do not get damped by photon viscosity, simply because the incompressible wavelike motions they induce, oscillate negligibly before recombination.

- As the photon mean-free-path grows, the fast compressible motions, induced by the magnetic field or due to existing "adiabatic" density fluctuations, are damped on scales smaller than the Silk scale, that is on scales $k^{-1}$ less than about $0.3 L_{S} \sim 0.3\left(l_{\gamma}(t) t\right)^{1 / 2}$. The comoving Silk damping scale, $L_{S}^{C}=L_{S} / a$, at any time $t$ (or temperature $T$ ) in the radiation era, is given by

$$
L_{S}^{C}(T) \approx 8.5 \times 10^{25}\left(\frac{T}{0.25 e V}\right)^{-3 / 2} f_{b}^{-1 / 2} \mathrm{~cm} .
$$


After matter domination, when the scale factor $a(t) \propto t^{2 / 3}$, the co-moving Silk scale is given by

$$
L_{S}^{C}(T) \approx 6 \times 10^{25}\left(\frac{T}{0.25 e V}\right)^{-5 / 4} h^{-1 / 2} f_{b}^{-1 / 2} c m
$$

- By contrast, the evolution of non-linear Alfvén wave modes depends on the ratio $D / \omega_{0}$. Non-linear Alfvén wave modes which enter the Hubble radius when the temperature of the universe $T>2.5 \mathrm{eV}$, do so when $D / \omega_{0}<1$, and the mode is then initially in the damped oscillatory regime. But the photon mean-free-path grows as $a^{3}$ while wavelengths grow as $a$, and soon tangles on some scale are in the overdamped regime, with $D / \omega_{0}>1$. For a given scale, $k^{-1}$, this happens at a time $\tau_{O D}$, when the temperature of the universe drops to $T<T_{O D}$, where

$$
T_{O D}=10.8 \mathrm{eV} \times B_{-9}^{-1 / 2}\left(\frac{k^{-1}}{1 M p c}\right)^{-1 / 2} f_{b}^{-1 / 2} .
$$

So, tangles on smaller scales not only enter the Hubble radius at an earlier epoch, but are overdamped at an earlier epoch.

- While the Alfvén mode is in the damped oscillatory regime, its evolution can be described by Eq. (4.17), and so all the modes damp by a factor of order $\exp \left(-\int^{\tau}(D / 2)\right) \sim$ $\exp \left[-(3 / 4)\left(4 k_{p}^{2}(\tau) L_{S}^{2}(\tau) / 45\right)\right]$. This is almost the same as the Silk damping factor, for the usual baryon-photon sound waves. Hence, all modes which are smaller than the Silk scale will get significantly damped by photon viscosity before the non-linear Alfvén mode enters the overdamped regime. The largest comoving scale, say $k^{-1}=k_{\text {osc }}^{-1}$, which gets damped while the mode is in the damped oscillatory regime, can be estimated by equating $\left[k_{\text {osc }} L_{S}^{C}\left(T_{O D}, k_{o s c}\right)\right] / 15=1$. This gives

$$
k_{\text {osc }}^{-1} \approx 4.4 \times 10^{-7} B_{-9}^{3} f_{b} M p c .
$$

- In the damped oscillatory regime, a mode which starts initially from rest $(\dot{f}=0)$, can be described by the solution $f(\tau) \approx f_{0} \cos \left[\chi\left(\tau-\tau_{e}\right)\right] \exp \left[-\int(D / 2)\right]$. Even modes which damp negligibly in this above regime, will oscillate by a phase of order

$$
\chi\left(\tau_{O D}\right) \sim k V_{A} \tau_{O D} \approx 4.14 \times 10^{-3} B_{-9}^{3 / 2}\left(\frac{k^{-1}}{1 M p c}\right)^{-1 / 2} f_{b}^{1 / 2}
$$

when the mode enters the overdamped regime. Modes with $\chi\left(\tau_{O D}\right)>1$ will acquire a large $\dot{f}$ by the time they enter the overdamped regime and will be strongly damped. This will happen for any mode with $k^{-1}=k_{O D}^{-1}<1.7 \times 10^{-5} B_{-9}^{3} f_{b} \mathrm{Mpc}$.

- It turns out that, for the Alfvén-type modes with $k^{-1}>5 k_{O D}^{-1}$, which enter the Hubble radius with zero initial velocity, there is negligible further damping while the mode is in the overdamped regime. When these modes enter the overdamped regime, with $\omega_{0} / D=1$, we find that they do not have sufficient velocity $(\dot{f})$ for friction to be 
important. With increasing time, the velocity grows, $D$ increases, and $\omega_{0} / D$ decreases. Eventually, the mode enters the terminal-velocity regime, at say a time $\tau_{T}$, where friction balances the Lorentz force (see Section IV). This happens roughly when $\tan \left(\chi\left(\tau_{T}\right)\right) \approx\left(\omega_{0} / D\left(\tau_{T}\right)\right)<<1$ and $f\left(\tau_{T}\right) \sim f_{0}$. In the terminal velocity regime, we have from Eq. (4.21), that

$$
f(\tau)=f\left(\tau_{T}\right) \exp \left[-\int_{\tau_{T}}^{\tau} \frac{\omega_{0}^{2}}{D} d \tau\right] \approx f\left(\tau_{T}\right) \exp \left[-0.9 \times 10^{-4}\left(\frac{T_{T}-T}{0.25 \mathrm{eV}}\right) B_{-9}^{2}\right] .
$$

For all modes with $l_{B}>5 l_{O D}$, one finds that there is no significant damping.

- When the photon mean-free-path increases above the wavelength of a given mode, one enters the free-streaming regime. As we discussed in Section VI, the further evolution of the Alfvén-type mode depends on the whether magnetic pressure at this time dominates the fluid pressure, or vice versa. This is determined by the ratio $B / B_{\text {crit }}$. For the case of $B<<B_{\text {crit }}$, one can again treat the evolution as incompressible. The resulting Alfvén modes are already in the overdamped regime when they start to free-stream and their evolution is governed by Eq. (6.9),

$$
\bar{f}(\tau)=\bar{f}\left(\tau_{f}\right) \exp -\left[\int_{\tau_{f}}^{\tau} \frac{\bar{\omega}_{0}^{2}}{\bar{D}} d t\right]=\bar{f}\left(\tau_{f}\right) \exp -V_{A}^{2}\left[\int_{t_{f}}^{t} k_{p}^{2}(t) l_{\gamma}(t) d t\right] .
$$

Hence, $\bar{f}(\tau)=\bar{f}\left(\tau_{f}\right) \exp \left(-k^{2} / k_{f s}^{2}\right)$, where the free-streaming damping scale $k_{f s}^{-1}$ is given by

$$
k_{f s}^{-2}=V_{A}^{2} \int_{t_{f}}^{t} \frac{l_{\gamma}(t) d t}{a^{2}(t)}
$$

Modes with a scale for the magnetic field $k^{-1}<k_{f s}^{-1}$, get damped significantly during the free streaming evolution. We see that the damping in this regime is similar to Silk damping, except that the usual Silk damping integral within the exponential (cf. Eq. (5.14) ) is multiplied by an extra factor of $(15 / 2) V_{A}^{2}<<1$. For the linearised modes where the wave vector makes an angle $\theta$ to the zero-order field, one has to replace $V_{A}$ by $V_{A} \cos \theta$. After recombination, the viscous damping is subdominant, compared to expansion damping (since $l_{\gamma}$ exceeds the Hubble radius), and so can be neglected. So the largest scale to be damped is found by evaluating $k_{f_{s}}^{-1}$ at the recombination redshift. Assuming that the universe is matter dominated at recombination, we get $k_{f s}^{-1} \approx(3 / 5)^{1 / 2} V_{A} L_{S}^{C}\left(t_{r}\right)$. Hence, the damping scale is of order the Alfvén velocity times the Silk scale. The largest wavelength mode to be damped, say $L_{D}^{A} \equiv 2 \pi k_{f s}^{-1}\left(t_{r}\right)$ is given by,

$$
L_{D}^{A}=2 \pi\left(\frac{3}{5}\right)^{1 / 2} V_{A} \frac{L_{S}\left(t_{r}\right)}{a\left(t_{r}\right)} \approx 1.1 \times 10^{23} B_{-9} f_{b}^{-1 / 2} h^{-1 / 2} \mathrm{~cm} .
$$

- For $B>B_{\text {crit }}$, we noted in Section VI that the evolution becomes compressible, and gravitationally unstable for scales larger than the magnetic Jeans length, $\lambda_{J}$. On such 
scales, we showed that a field with $B_{-9} \sim 1$, is needed to produce a density perturbation large enough to significantly affect galaxy formation. We did not treat the evolution on scales smaller than the magnetic Jeans length in any detail, although the governing equations are given in Appendix B (cf. Eq. (B8)). The solution of these equations, as we noted in Section VII, is complicated by the presence of an inhomogeneous zero-order magnetic field. Nevertheless, we expect that fast compressible motions on scales smaller than $\lambda_{J}$ will drive oscillations close to the baryonic Alfvén frequency, and will be initially overdamped by the action of photon viscosity, in the pre-recombination era. The damping scale for such motions will then be similar to $k_{f s}^{-1}$, as deduced above. For modes similar to the slow magneto-sonic wave, we expect that for $B>>B_{c r i t}$, the phase velocity will be roughly equal to $c_{b}$, the baryon sound speed, and the corresponding damping scale will be $k_{f s}^{-1}$ with $V_{A}$ replaced by $c_{b}$. These expectations are borne out by the linearised calculations of JKO, although they ignore the inhomogeneous nature of the zero-order magnetic field. (For a general tangled zero order field, the counterpart of the slow wave may not be easy to excite). Clearly, more detailed computations are needed to get the exact damping scales, in this case.

- One can consider the damping due to neutrino viscosity in the early universe [23], in an exactly analogous manner to the Silk damping effects treated in detail above. We briefly describe below some of the consequences of damping due to neutrinos. These damping effects are largest around the time of neutrino decoupling, at a temperature of $T=T_{\nu} \sim 1 \mathrm{MeV}$. During this epoch, the number density of weakly interacting particles is $n_{W} \sim T^{3}$ (cf. [19], [18]). The cross-section for interaction with neutrinos, is typically the weak interaction cross-section $\sigma_{W} \sim G_{F}^{2} T^{2}$, where $G_{F}$ is the Fermi constant. The neutrino mean-free-path is then given by

$$
l_{\nu}=\frac{1}{\sigma_{W} n_{W}} \approx 1.4 \times 10^{11} \mathrm{~cm}\left(\frac{T}{M e V}\right)^{-5} .
$$

Note that $l_{\nu}$ becomes comparable to the Hubble radius, $H^{-1}=2 t \approx 4.4 \times$ $10^{10} \mathrm{~cm}(\mathrm{~T} / \mathrm{MeV})^{-2}$, at $T \sim 1 \mathrm{MeV}$. The comoving damping scale for fast modes, say $L_{S}^{C \nu}$, due to neutrino viscosity, can be derived in exactly analogous manner to the Silk damping scale, derived in Section V. We get $L_{S}^{C \nu}=\left[t\left(T_{\nu}\right) l_{\nu}\left(T_{\nu}\right)\right]^{1 / 2} / a\left(T_{\nu}\right) \sim$ $l_{\nu}\left(T_{\nu}\right) / a\left(T_{\nu}\right) \approx 0.9 \times 10^{20} \mathrm{~cm}$. As with the damping by photon viscosity, the maximum damping of Alfvén (or the slow) modes occur when they enter the free-streaming regime. We analyse the damping of the non-linear Alfvén wave in the neutrino freestreaming regime, in Appendix C. We show there that damping in this regime is similar to Silk damping except that the usual Silk damping integral within the exponential (cf. Eq. (5.14) ) is multiplied by an extra factor of $(15 / 2) V_{A \nu}^{2}<<1$, and $l_{\gamma}$ is replaced by $l_{\nu}$. Here, $V_{A \nu}$ is the Alfvén velocity defined in terms of the conserved, neutrino energy density $\rho_{\nu}\left(T_{\nu}\right) a^{4}\left(T_{\nu}\right)$. Since $l_{\nu} \propto T^{-5} \propto t^{5 / 2}$ in the radiation-dominated epoch, we can estimate the largest wavelength Alfvén mode that is appreciably damped, by neutrino viscosity, in the free streaming regime. This comoving wavelength is given by $L_{D \nu}^{A} \sim 2 \pi(2 / 5)^{1 / 2} V_{A \nu} L_{S}^{C \nu} \sim 10^{17} B_{-9} \mathrm{~cm}$.

We can generalise these results given any initial spectrum of magnetic inhomogeneities, by replacing $V_{A}$ by a suitably averaged scale-dependent $V_{A}\left(k^{-1}\right)$. 


\section{CONCLUSIONS}

We have considered the evolution and viscous damping of cosmic magnetic fields in the early universe in detail. Using the fact that the fluid, electromagnetic, and shear viscous energy-momentum tensors are all conformally invariant, we showed in Section II that the MHD equations in the FRW universe, including viscous effects, can be transformed into their special-relativistic counterpart when the metric perturbations from inhomogeneous motions are small. This enabled us to transform known non-linear Alfvén-wave solutions, from flat spacetime, into the expanding FRW universe.

We considered in detail the evolution and damping of these modes in various regimes. First, on galactic scales or larger, the Alfvén mode oscillates negligibly before recombination, for magnetic field strengths, $B_{-9}<1$ (or a present day magnetic field $B<10^{-9}$ Gauss). So there is then no question of strong "Silk" damping of these modes, due to photon viscosity, as occurs for compressional baryon-photon oscillations. Furthermore, Alfvén waves with small enough wavelength, which can oscillate appreciably before recombination, become overdamped. In this case, the longest wavelength which suffers appreciable damping by photon viscosity, has a scale $k^{-1} \sim V_{A} L_{S}^{C}$, where $L_{S}^{C}$ is the usual comoving Silk scale. Since the Alfvén speed is $V_{A} \sim 3.8 \times 10^{-4} B_{-9}<<1$, only comoving wavelengths smaller than $10^{23} B_{-9} \mathrm{~cm}$ suffer appreciable damping. We also briefly considered analogous results for damping of very small scales by neutrino viscosity following neutrino decoupling at $t \sim 1 s$.

After recombination, the fluid pressure drops enormously, roughly by the baryon to photon ratio. The Lorentz force due to a tangled magnetic field associated with an initially Alfvén-like mode before recombination can seed gravitationally unstable compressional perturbations after recombination, provided the field is strong enough and tangled enough on scales larger than the magnetic Jeans length, $\lambda_{J}$. We examined the post-recombination evolution of scales larger than $\lambda_{J}$ in section VII, including the effect of a passive dark matter component, and showed that magnetic fields with $B_{-9} \sim 1$ are needed to impact significantly on galaxy formation. The evolution equations for perturbations with scales smaller than $\lambda_{J}$ are derived in Appendix B, but their solution is much more complicated and will be examined elsewhere.

Magnetic fields with $B_{-9} \sim 1$, may be constrained by observations of quasar rotation measures [21]. The fluid velocities induced by the tangled field, oscillating as an Alfvén mode in the pre-recombination era, can also produce small angular scale anisotropies in the microwave background, through the Doppler effect [22]. This may provide another constraint on such fields. The dissipation of magnetic fields due to neutrino or photon viscosity will also leave an imprint on the spectrum of neutrinos and photons respectively. The neutrino spectrum could be probed using nucleosynthesis and there already exist strong limits on the spectral distortions of the microwave background. Scenarios of galaxy formation that appeal to strong enough magnetic fields of order $10^{-9}$ Gauss are potentially testable. We hope to return to a further consideration of some of these observational issues in future work.

\section{ACKNOWLEDGMENTS}

KS was supported by a PPARC Visiting Fellowship at the Astronomy Centre, University of Sussex. He thanks the staff there for warm hospitality. JDB is supported by a PPARC 
Senior Fellowship.

\section{APPENDIX A: MAXWELL'S EQUATION IN THE"LAB" FRAME}

In the main text we defined the electric field $\mathbf{E}^{*} \equiv\left(E^{* 1}, E^{* 2}, E^{* 3}\right)$ and magnetic field $\mathbf{B}^{*} \equiv\left(B^{* 1}, B^{* 2}, B^{* 3}\right)$ in the starred metric by

$$
F^{* 0 i}=E^{* i} \quad F^{* 12}=B^{* 3} \quad F^{* 23}=B^{* 1} \quad F^{* 31}=B^{* 2} .
$$

This can be written in more compact way in term of a four-vector electric field $E_{\mu}^{*}$ and magnetic field $B_{\mu}^{*}$ as

$$
B_{\mu}^{*}=\frac{1}{2} \epsilon_{\mu \nu \rho \lambda}^{*} V^{* \nu} F^{* \rho \lambda} ; \quad E_{\mu}^{*}=F_{\mu \nu}^{*} V^{* \nu}
$$

where $V^{* \mu} \equiv[1,0,0,0]$ is the four-velocity of fundamental observers at rest in the starred metric frame. We have also used the Levi-Civita tensor $\epsilon_{\mu \nu \rho \lambda}^{*}=\sqrt{-g^{*}} \mathcal{A}_{\mu \nu \rho \lambda}$, with $\mathcal{A}_{\mu \nu \rho \lambda}$, the totally antisymmetric symbol such that $\mathcal{A}_{0123}=1$ and \pm 1 for any even or odd permutations of $(0,1,2,3)$. Note that the four-vectors $B_{\mu}^{*}$ and $E_{\mu}^{*}$ have purely spatial components and $E_{i}^{*}=E^{* i}, B_{i}^{*}=B^{* i}$ for the spatial components of the field 4-vector. First, let us transform the electric and magnetic four-vectors to the unstarred (FRW) conformal frame, and denote the resulting electric and magnetic four-vectors by $E_{\mu}^{\prime}$ and $B_{\mu}^{\prime}$, respectively. Making use of the conformal transformation properties,

$$
F^{* \mu \nu}=\Omega^{-4} F^{\mu \nu} ; \quad \epsilon_{\mu \nu \rho \lambda}^{*}=\Omega^{4} \epsilon_{\mu \nu \rho \lambda} ; \quad V^{* \nu}=\Omega^{-1} V^{\nu},
$$

we get

$$
B_{\mu}^{\prime}=\Omega B_{\mu}^{*}=\frac{1}{a} B_{\mu}^{*} ; \quad E_{\mu}^{\prime}=\Omega E_{\mu}^{*}=\frac{1}{a} E_{\mu}^{*}
$$

We now make a coordinate transformation to the FRW proper "Lab" co-ordinates $(t, \mathbf{r})$ defined by $d t=a d \tau, d \mathbf{r}=a d \mathbf{x}$. Denote the co-ordinate-transformed, "Lab" electric and magnetic four-vectors by $E_{\mu}$ and $B_{\mu}$, respectively. We have

$$
B_{\mu}=\frac{1}{a} B_{\mu}^{\prime}=\frac{1}{a^{2}} B_{\mu}^{*} ; \quad E_{\mu}=\frac{1}{a} E_{\mu}^{\prime}=\frac{1}{a^{2}} E_{\mu}^{*} .
$$

Note that these 4-vectors are also purely spatial. Similarly, we can define the current density in the Lab frame by $J_{L}^{\mu}=a J^{\mu}=a \Omega^{4} J^{* \mu}=a^{-3} J^{* \mu}$. Now, denote the spatial components $B_{\mu}, E_{\mu}$ and $J_{L}^{\mu}$ by the spatial 3 -vectors $\mathbf{B}, \mathbf{E}$ and $\mathbf{J}$ respectively. Then, in terms of these spatial vectors, we have

$$
\mathbf{B}=\frac{\mathbf{B}^{*}}{a^{2}} ; \quad \mathbf{E}=\frac{\mathbf{E}^{*}}{a^{2}} ; \quad \mathbf{J}=\frac{\mathbf{J}^{*}}{a^{3}}
$$

The four-current density will also have a time component, the charge density $\rho_{q}=J_{L}^{0}=$ $\left(J^{* 0} / a^{3}\right)$. Using the Maxwell equations, (3.4), in the starred metric, one can write the Maxwell equations in terms of these "Lab" fields. We have 


$$
\begin{gathered}
\nabla \times\left(a^{2} \mathbf{B}\right)=4 \pi a^{3} \mathbf{J}+\frac{\partial\left(a^{2} \mathbf{E}\right)}{\partial \tau} ; \quad \nabla \cdot \mathbf{B}=0 \\
\nabla \times\left(a^{2} \mathbf{E}\right)=-\frac{\partial\left(a^{2} \mathbf{B}\right)}{\partial \tau} ; \quad \nabla \cdot\left(a^{2} \mathbf{E}\right)=4 \pi a^{3} \rho_{q} .
\end{gathered}
$$

The non-relativistic limit of Ohm's law in terms of the "Lab" fields is simply

$$
\mathbf{E}+\mathbf{v} \times \mathbf{B}=\frac{\mathbf{J}}{\sigma} .
$$

In the ideal limit, the "Lab" magnetic field then satisfies the induction equation

$$
\frac{\partial\left(a^{2} \mathbf{B}\right)}{\partial t}=\frac{1}{a} \frac{\partial\left(a^{2} \mathbf{B}\right)}{\partial \tau}=\frac{1}{a} \nabla \times\left[\mathbf{v} \times\left(a^{2} \mathbf{B}\right)\right] .
$$

So, when $\mathbf{v}=0$, we have $\mathbf{B} \propto a^{-2}$, a result which is intuitively expected for the "Lab" magnetic field due to flux freezing in the expanding universe. It is also of interest to express the Euler equation (3.3) for the fluid in the radiation-dominated era, in terms of the "Lab" fields. We obtain

$$
\begin{aligned}
\frac{1}{a^{4}} \frac{\partial}{\partial t}\left[(\rho+p) a^{4} \mathbf{v}\right]+\frac{(\mathbf{v} \cdot \nabla)}{a}[(\rho+p) \mathbf{v}] & +\frac{\mathbf{v}}{a} \nabla \cdot[(\rho+p) \mathbf{v}] \\
& =-\frac{1}{a} \nabla p+\mathbf{J} \times \mathbf{B}+\frac{\eta}{a^{2}}\left[\nabla^{2} \mathbf{v}+\frac{1}{3} \nabla(\nabla \cdot \mathbf{v})\right] .
\end{aligned}
$$

\section{APPENDIX B: GENERALISED LINEAR PERTURBATION THEORY IN THE FREE-STREAMING AND POST-RECOMBINATION REGIMES}

Begin with the linearised evolution equations for the baryonic fluid, including the effects of the magnetic field and gravity. We have

$$
\begin{gathered}
\frac{\partial \mathbf{v}}{\partial t}+H(t) \mathbf{v}=-\frac{1}{a \rho_{b}} \nabla p_{1}+\frac{1}{\rho_{b}} \mathbf{J} \times \mathbf{B}-\frac{1}{a} \nabla \phi-\frac{4 \rho_{\gamma}}{3 \rho_{b}} n_{e} \sigma_{T} \mathbf{v} \\
\frac{\partial \delta_{b}}{\partial t}+\frac{1}{a} \nabla \cdot \mathbf{v}=0 . \\
\nabla^{2} \phi=4 \pi G a^{2} \delta \rho_{T}=4 \pi G a^{2}\left[\rho_{b} \delta_{b}+\rho_{c} \delta_{c}\right]
\end{gathered}
$$

Here $p_{1}=c_{b}^{2} \rho_{b} \delta_{b}$ is the perturbed pressure. We take $\mathbf{B}=\left[\mathbf{B}_{0}(\mathbf{x})+\mathbf{b}(\mathbf{x})\right] / a^{2}$, with $|\mathbf{b}|<<$ $\left|\mathbf{B}_{0}\right|$. The linearised induction equation, (A10), then becomes

$$
\frac{\partial \mathbf{b}}{\partial t}=\frac{1}{a} \nabla \times\left[\mathbf{v} \times \mathbf{B}_{0}\right] ; \quad \nabla \cdot \mathbf{b}=0 .
$$


Suppose the perturbation is described in terms of the perturbed position of the baryonic component by $\delta \mathbf{x}_{b}=\xi(\mathbf{x}, \tau)$. The perturbed velocity is $\mathbf{v}=a(\partial \xi / \partial t)$. An integration of the perturbed continuity equation (B2) and induction equation (B4) then gives

$$
\delta_{b}=-\nabla . \xi \quad \mathbf{b}=\nabla \times\left[\xi \times \mathbf{B}_{0}\right] .
$$

Similarly, one can define the perturbed comoving position of the cold dark matter component by $\delta \mathbf{x}_{c}=\xi_{c}(\mathbf{x}, \tau)$. An integration of the perturbed continuity equation for this component gives $\delta_{c}=-\nabla \cdot \xi_{c}$. The Poisson equation (B3) for the potential can then be integrated once to give

$$
-\frac{1}{a} \nabla \phi=4 \pi G a\left[\rho_{b} \xi+\rho_{c} \xi_{c}\right] .
$$

The perturbed pressure-gradient term can be written as

$$
-\frac{1}{a \rho_{b}} \nabla p_{b}=\frac{c_{b}^{2}}{a} \nabla(\nabla \cdot \xi) .
$$

Using Eq. (B5), (B6) and (B7), the perturbed Euler equation becomes

$$
\begin{aligned}
\frac{\partial^{2} \xi}{\partial t^{2}}+\left[2 H+\frac{4 \rho_{\gamma}}{3 \rho_{b}} n_{e} \sigma_{T}\right] \frac{\partial \xi}{\partial t}=\frac{c_{b}^{2}}{a^{2}} \nabla(\nabla . \xi)+ & 4 \pi G\left[\rho_{b} \xi+\rho_{c} \xi_{c}\right]+\frac{\left(\nabla \times \mathbf{B}_{0}\right) \times \mathbf{B}_{0}}{\left(4 \pi \rho_{b} a^{3}\right) a^{3}} \\
& +\frac{\left[\nabla \times\left(\nabla \times\left[\xi \times \mathbf{B}_{0}\right]\right)\right] \times \mathbf{B}_{0}}{\left(4 \pi \rho_{b} a^{3}\right) a^{3}} \\
& +\frac{\left(\nabla \times \mathbf{B}_{0}\right) \times\left[\left(\nabla \times\left[\xi \times \mathbf{B}_{0}\right)\right]\right.}{\left(4 \pi \rho_{b} a^{3}\right) a^{3}} .
\end{aligned}
$$

This linear equation describes the gravitationally unstable evolution. and damping of linearised MHD modes in the free-streaming and post-recombination regime. However, one cannot perform a simple Fourier analysis of this equation, since $\mathbf{B}_{0}$ is also a function of $\mathbf{x}$. In the main text we have simplified the equation by neglecting the last two terms. While this approximation is likely to be valid for large-scale modes at early times, it will break down once the distortion of the magnetic field due to the motions become significant. We hope to return to the study of this equation elsewhere.

\section{APPENDIX C: VISCOUS DAMPING DUE TO NEUTRINOS IN THE FREE-STREAMING REGIME}

Modes whose wavelengths become longer than the neutrino mean-free-path, enter the neutrino free-streaming regime. The evolution and damping of non-linear Alfvén wave solutions can be examined in this regime, analogous to the case of photon-free streaming, treated in section VI. First the viscous force due to coupling of the relativistic plasma and the neutrinos is given by [23]

$$
\mathbf{F}_{D}^{\nu}=-\frac{4}{3} n_{W} \sigma_{W} \rho_{\nu} \mathbf{v}=\frac{4}{3} \rho_{\nu} \frac{\mathbf{v}}{l_{\nu}} .
$$


Here, $\rho_{\nu}$ is the energy density of neutrinos, and we have replaced $\left(n_{W} \sigma_{W}\right)^{-1}$ by the neutrino mean-free-path $l_{\nu}$. We can also use the form of the Euler and induction equations, (as derived in Appendix A); except that the viscous force is as given by Eq. (C1) and the fluid inertia/pressure does not include the neutrino contributions. Following the ideas of Section IV and VI, we look again at non-linear Alfvén modes with $\mathbf{B}=\left(\mathbf{B}_{0}+\mathbf{b}\right) / a^{2}$, where $\mathbf{B}_{0}=B_{0} \hat{\mathbf{z}}$, with $B_{0}=$ constant, $\mathbf{b}=\mathbf{n} g(\tau) e^{i k z}$ and $\mathbf{v}=\mathbf{n} \bar{v}_{g}(\tau) e^{i k z}$, with $\mathbf{n}$ perpendicular to $\hat{\mathbf{z}}$. Recall that $|\mathbf{b}|$ is not necessarily small compared to $\left|\mathbf{B}_{0}\right|$. Also, since the universe is still radiation dominated at the epochs near neutrino decoupling, with $p>>B^{2} /(8 \pi)$, we can treat the motions as nearly incompressible. The Euler equation gives

$$
\frac{4 \rho_{R} a^{4}}{3} \frac{\partial \mathbf{v}}{\partial \tau}=\frac{B_{0}}{4 \pi} \frac{\partial \mathbf{b}}{\partial z}-\frac{4}{3} \rho_{\nu} a \frac{\mathbf{v}}{l_{\nu}}
$$

where $\rho_{R}$ is the fluid density, excluding the neutrino contribution. The induction equation gives

$$
\frac{\partial \mathbf{b}}{\partial \tau}=B_{0} \frac{\partial \mathbf{v}}{\partial z}
$$

These can be combined to give an equation for the evolution of $g(\tau)$

$$
\frac{d^{2} g}{d \tau^{2}}+D_{\nu} \frac{d g}{d \tau}+\omega_{\nu}^{2} g=0
$$

where

$$
\omega_{\nu}=k V_{A R} ; \quad D_{\nu}=\frac{a}{l_{\nu}}\left(\frac{4 \rho_{\nu}}{3 \rho_{R}}\right) \propto \tau^{-4}
$$

Here, $V_{A R}^{2}=3 B_{0}^{2} /\left(16 \pi \rho_{R} a^{4}\right)=$ constant, is the Alfvén velocity defined with the inertia contributed by $\rho_{R}$.

The evolution of this non-linear Alfvén mode depends once again on the relative strengths of the damping and driving terms. When modes enter the neutrino free-streaming regime, the Alfvén waves are again strongly overdamped, with $D_{\nu} / \omega_{\nu}>>1$, for $B_{-9}<1$. One can again apply the terminal-velocity approximation of Section IV in the overdamped regime, once $d g / d \tau$ has adjusted itself to the "zero acceleration" solution. Then, $g$ is given by

$$
g(\tau)=g\left(\tau_{i}\right) \exp \left(-\int_{\tau_{i}}^{\tau} k^{2} V_{A \nu}^{2} \frac{l_{\nu}}{a} d \tau\right)
$$

where

$$
V_{A \nu}^{2}=\frac{B_{0}^{2}}{4 \pi\left(4 \rho_{\nu} a^{4} / 3\right)}
$$

is the Alfvén velocity defined in terms of the conserved neutrino energy density $\rho_{\nu} a^{4}$. We see that the damping of these Alfvén modes by neutrino viscosity is similar to Silk damping except that the usual Silk damping integral within the exponential (cf. Eq. (5.14)) is multiplied by an extra factor of $(15 / 2) V_{A \nu}^{2}<<1$, and $l_{\gamma}$ is replaced by the neutrino meanfree-path $l_{\nu}$. 
*Presently at: Max Plank Institute for Astrophysics, Karl Schwarzschild Strasse 1, 85748 Garching, Germany. On leave from National Centre for Radio Astrophysics, TIFR Pune, Poona University Campus, Ganeshkhind, Pune 411 007, India. 


\section{REFERENCES}

[1] Moffat, H. K., Magnetic Field Generation in Electrically Conducting Fluids, Cambridge University Press, Cambridge, (1978); Krause, F. \& Radler, K.-H., Mean-Field Magnetohydrodynamics and Dynamo Theory, Pergamon Press, Oxford, (1980); Ruzmaikin, A. A., Shukurov, A. M. \& Sokoloff, D. D., Magnetic Fields of Galaxies, Kluwer, Dordrecht (1988); Mestel, L., Stellar Magnetism, Oxford UP, Oxford, (1998); Beck, R., Brandenburg, A., Moss, D., Shukurov, A. \& Sokoloff, D., Ann. Rev. Astron. Astrophys., 34, 155 (1996).

[2] Vishniac, E.T., Ap. J. 446, 724 (1995); Rees, M. J., Quart. Jl. Roy. Astron. Soc. 28, 197 (1987); Rees, M.J., In Cosmical Magnetism, ed. Lynden-Bell, D., Kluwer, London, (1994) p155; Subramanian, K., Bull. Astr. Soc. Ind., 23, 481 (1995); Subramanian, K., Narasimha, D. \& Chitre, S.M., Mon. Not. R. astron. Soc. 271, L15 (1994), and references therein.

[3] Hogan, C. J., Phys. Rev. Lett., 51, 1488 (1983); Turner, M. S. \& Widrow, L. M., Phys. Rev. D, 30, 2743 (1988); Vachaspati, T., Phys. Lett. B, 265, 258 (1991). Ratra, B.,. Ap.J Lett., 391, L1 (1992); Gasperini, M., Giovannini, M. \& Veneziano, G., Phys. Rev. Lett. 75, 3796 (1995); Lemoine, D., \& Lemoine, M., Phys. Rev. D, 52, 1955 (1995); O. Törnkvist, hep-ph/9707513; K. Enqvist, astro-ph/9707300; Giovaninni, M., and Shaposhnikov, M.E., hep-ph/9710234; Joyce, M. and Shaposhnikov, M.E., Phys. Rev. Lett., 79, 1193 (1997).

[4] Cattaneo, F. \& Vainshtein, S. I., Ap.J., 376, L21 (1991); Vainshtein, S. \& Rosner, R., Ap.J., 376, 199 (1991); Kulsrud, R.M. \& Anderson, S.W., Ap.J., 396, 606 (1992); Brandenburg, A., In Lectures on Solar and Planetary Dynamos, ed. Proctor, M. R. E. \& Gilbert, A. D., Cambridge University Press, (1994), p117; Field, G. B., The Physics of the Interstellar Medium and Intergalactic Medium, ed. Ferrara et al., ASP Conf. Ser., 80, (1996), p1; Blackman, E., Phys. Rev. Lett., 77, 2694 (1996); Chandran, B., Princeton University Observatory preprint (1996) POPe-659; Cattaneo, F. \& Hughes, D. W., Phys. Rev E, 54, 4532 (1996); Subramanian, K., Mon. Not. R. astron. Soc. in press; astro-ph/9707280.

[5] Kulsrud, R., IAU Symp. 140: Galactic and Extragalactic Magnetic Fields, Reidel, Dordrecht, (1990), p527, and references therein.

[6] J.D. Barrow, P.G. Ferreira, and J. Silk, Phys. Rev. Lett. 78, 3610 (1997); J.D. Barrow, Phys. Rev. D 55, 7451 (1997).

[7] Silk, J., Ap. J., 151, 431 (1968).

[8] Jedamzik, K., Katalinic, V., and Olinto, A., (1996), astro-ph/9606080 v2

[9] Weinberg, S., Gravitation and Cosmology, Wiley, New York, (1972); G.F.R. Ellis, in General Relativity and Cosmology, ed. R.K. Sachs, Varenna Lectures in Physics, Academic, New York, (1971).

[10] Brandenburg, A., Enqvist, K., Olesen, P., Phys. Rev. D, 54, 1291 (1996).

[11] Liang, E. P. T., Ap. J., 211, 361 (1977).

[12] Parker, E. N., Cosmic Magnetic Fields, Clarendon Press, Oxford (1979).

[13] Landau, L. \& Lifshitz, E. M., Electrodynamics of Continuous Media, Pergamon Press, Oxford (1987).

[14] Walker, T. P., Steigman, G., Schramm, D. N., Olive, K. A. \& Kang, H.-S., Ap.J., 376, 51 (1991). 
[15] Steigman, G., Hata, N. \& Felten, J. E., astro-ph/9708016 and references therein.

[16] Wasserman, I., Ap.J., 224, 337 (1978)

[17] Peebles, P. J. E., Large Scale Structure of the Universe, Princeton University Press, Princeton N. J., (1980).

[18] Padmanabhan, T., Structure Formation in the Universe, Cambridge University Press, Cambridge (1993).

[19] Kolb, E. W. \& Turner, M. S., The Early Universe, Addison-Wesley, New York, (1990)

[20] Kaiser, N.,, Mon. Not. R. astron. Soc., 202, 1169 (1983).

[21] Kronberg, P. P., Rep. Prog. Phys., 57, 325 (1994)

[22] Subramanian, K. \& Barrow, J. D., 1998 (in preparation).

[23] Neutrino viscosity was first considered as a means of damping anisotropies and inhomogeneities by C.W. Misner, Nature 214, 40 (1967) and Phys. Rev. Lett. 19, 533 (1967). See also, J.D. Barrow, Nature 267, 117 (1977). The damping of very small scale irregularities by GUT scale transport processes is discussed by J.D. Barrow, Mon. Not. R. astron. Soc. 199, 45P (1982). 